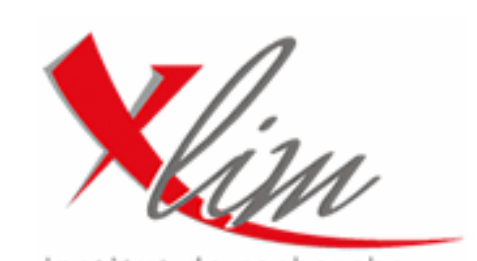

institut de recherche

\title{
Time-Domain Calibrated Measurements of Wideband Multisines Using a Large-Signal Network Analyzer
}

\author{
M. El Yaagoubi, G. Neveux, D. Barataud, T. Reveyrand, J.-M. Nebus, F. Verbeyst, \\ F. Gizard, J. Puech
}

\begin{abstract}
Published in
IEEE TRANSACTIONS ON MICROWAVE THEORY AND TECHNIQUES, VOL. 56, NO. 5, MAY 2008, pp. 1180-1192
\end{abstract}

(C) 2008 IEEE Personal use of this material is permitted. However, permission to reprint/republish or redistribute this material for advertising or promotional purposes or for creating new collective works for resale or redistribution to servers or lists, or to reuse any copyrighted component of this work in other works must be obtained from the IEEE. 


\title{
Time-Domain Calibrated Measurements of Wideband Multisines Using a Large-Signal Network Analyzer
}

\author{
Mohammed El Yaagoubi, Guillaume Neveux, Denis Barataud, Tibault Reveyrand, Jean-Michel Nebus, \\ Frans Verbeyst, Member, IEEE, Francis Gizard, and Jérôme Puech
}

\begin{abstract}
This paper presents a calibrated measurement technique that enables phase and magnitude measurements of wideband multisines. This study is based on the use of a large-signal network analyzer (LSNA) performing harmonic sub-sampling and a fine frequency grid $(20 \mathrm{MHz})$ comb generator calibrated using a high-frequency $50-\mathrm{GHz}$ equivalent-time sampling scope. This comb generator is used as a harmonic phase reference generator for the calibration of the LSNA.

The research reported here is applied to $L$-band multisine measurements, but it can be extended to higher microwave frequencies. The motivation of this study is to measure the multipactor phenomena effect in output RF multiplexers of satellite payloads.
\end{abstract}

Index Terms-Harmonic mixing, harmonic phase reference (HPR), large-signal network analyzer (LSNA), multisines, time-domain measurements.

\section{INTRODUCTION}

$\mathbf{T}$ IME-DOMAIN waveform measurements of microwave signals have been widely performed during the last decade for the characterization of nonlinear microwave devices. The need for a measurement setup having a good dynamic range (in the order of $60 \mathrm{~dB}$ ) along with a fast measurement time and a small time base distortion has led to the use of large-signal network analyzers (LSNAs) instead of sampling scopes. LSNA measurements are based on a sub-sampling procedure. Using this approach, microwave signals up to $50 \mathrm{GHz}$ can be frequency translated and compressed within a $10-\mathrm{MHz}$ IF bandwidth and then digitized using high dynamic-range analog-to-digital converters (ADCs).

To perform accurate LSNA measurements, one must first ensure that different RF frequencies do not fold down to the same IF frequencies during the sampling conversion. Next,

Manuscript received August 1, 2007; revised October 15, 2007. This work was supported in part by the Centre National d'Etudes Spatiales (CNES) under Contract R-S05/TC-0001-011-XLIM, by the Centre National d'Etudes Spatiales (CNES), and by the Information Society Technologies Programme of the European Union (EU) under Contract IST-1-507893-NOE.

M. El Yaagoubi, G. Neveux, D. Barataud, T. Reveyrand, and J.-M. Nebus are with the XLIM, Unité Mixte de Recherche (UMR), University of Limoges, 87060 Limoges, France (e-mail: guillaume.neveux@xlim.fr; denis. barataud@xlim.fr; tibault.reveyrand@xlim.fr; jean-michel.nebus@unilim.fr).

F. Verbeyst is with NMDG NV, B-2880 Bornem, Belgium (e-mail: frans.verbeyst@nmdg.be).

F. Gizard and J. Puech are with the Centre National d'Etudes Spatiales (CNES), 31401 Toulouse, France (e-mail: francis.gizard@cnes.fr; jerome.puech@cnes.fr).

Color versions of one or more of the figures in this paper are available online at http://ieeexplore.ieee.org. a three-step calibration procedure is needed in order to get accurate time-domain waveforms. It consists of a conventional short-open-load-thru (SOLT), thru-reflect-line (TRL), or linereflect-reflect-match (LRRM) relative calibration, an absolute power calibration using a power meter and an absolute phase calibration using a harmonic phase reference (HPR) generator. The phase calibration is a key point of the calibration procedure [1] and is problematic in the case of wideband modulated signal measurements. This phase-calibration procedure is the main focus of this paper. The aim of this phase calibration is to determine the phase distortion introduced by the LSNA. The phase calibration is required to determine the group-delay variations of each measurement channel. For that purpose, an HPR generator must be used. The commercially available HPR generator supplied with the Maury LSNA (Model MT4463A) is a comb generator driven by a sinusoidal input signal having a frequency that can be set between $600 \mathrm{MHz}-1.2 \mathrm{GHz}$. This reference generator produces significant harmonic components at its output up to $50 \mathrm{GHz}$. It is characterized using a $50-\mathrm{GHz}$ equivalent-time scope calibrated using either the nose-to-nose technique [2], [3] or the electro-optic sampling technique [4], which is a metrology technique traceable to the NIST. Phase relationships between harmonic spectral components generated by the HPR generator are given in a calibration data file.

Today, time-domain LSNA measurements of nonlineardevices driven by continuous wave (CW) signals are well established. They have contributed to the experimental optimization of operation classes of transistors for high-efficiency amplifier designs [5]-[7]. LSNA measurements using high-impedance probes enable voltage measurements at internal nodes of monolithic microwave integrated circuits [8]-[10]. RF pulsed operation is also interesting for the characterization and modeling of high-power transistors, where thermal aspects pose additional challenges [11]. Mixer measurements have also been reported [12], [13]. In the previously mentioned research, the spectral components of the signals (carriers and harmonics or mixing products) must be set on a frequency grid having a minimum frequency step of $600 \mathrm{MHz}$ and a maximum frequency step of $1.2 \mathrm{GHz}$. This is related to the frequency grids used for the calibration of the commercially available HPR generator.

Multisines having frequency spacing between a few tens of kilohertz and approximately $20 \mathrm{MHz}$ are useful for the characterization of nonlinear devices. A quite simple two-tone signal is useful for linearity characterization of power amplifiers with memory [14]. It can also be useful for the fast load-pull 
measurements of power transistors [15]. Multisines are also useful for the purpose of behavioral modeling [16]. In that particular case, the required frequency grid is much lower than $600 \mathrm{MHz}$ and must be accommodated to the frequency spacing of multisine test signals. Using the LSNA technique for multisine measurements, two main difficulties must be carefully examined.

First, a multisine phase calibrated generator must be used for the phase calibration of the LSNA. Solutions have been proposed for narrow-bandwidth multisine reference generators [17], [18]. However, wideband communication systems like satellite communication systems may require a bandwidth up to $250 \mathrm{MHz}$.

Second, when multisine RF signals have a bandwidth larger than $10 \mathrm{MHz}$, multiple harmonic mixing products occur in the down-converter stage and fall within the IF bandwidth of the instrument. This results in a quite complex frequency compressed IF spectrum. A way to accurately determine phase relationships between RF spectral components and IF scrambled spectral components has been proposed in [19]. It consists in measuring several narrow bandwidths, which are carefully interleaved. Applying spectral stitching [20], phase relationships between tones of adjacent bandwidths can be extracted. Nevertheless, this solution is not very straightforward and suffers from errors such that the uncertainty of the phase increases when moving toward both edges of the modulation frequency range.

In this section, we present a more straightforward technique, which is based on the use of a new HPR generator having a $20-\mathrm{MHz}$ frequency grid. This reference phase generator has been calibrated by NMDG using an equivalent-time sampling scope. It can be argued that a finer frequency grid would have been better, but we found that $20 \mathrm{MHz}$ was a good compromise for our application, which is the characterization of wideband $80-\mathrm{MHz}$ multisines. This signal is very appropriate to study the multipactor effect in satellite payloads. As a consequence, in this paper, we propose to use a new HPR generator, which consists of a multisine covering the entire bandwidth $(20$ $\mathrm{MHz}-4 \mathrm{GHz}$ ) with a $20-\mathrm{MHz}$ frequency spacing. The proposed technique is similar to the one reported in [21] and [22]. The technique presented here is a sampler-based technique, while the technique reported in [21] and [22] is a mixer-based technique. In mixer-based techniques, the different tones of the signals are sequentially measured. This technique requires an addition fifth channel connected to a harmonic phase generator for phase relationship measurements [7]. In sampler-based techniques, all the tones of interest are measured simultaneously.

In Section II, we give an overview of the basic problems encountered when wideband multisines are measured using the LSNA. The proposed approach for wideband multisine measurements is then explained.

Section III is dedicated to the description of the specific new HPR generator and its use for LSNA calibration. The HPR generator calibration procedure is also explained.

In Section IV, some measurement results are shown and discussed. To conclude, the future use of our calibrated setup is highlighted for multipactor characterization in satellite payloads.

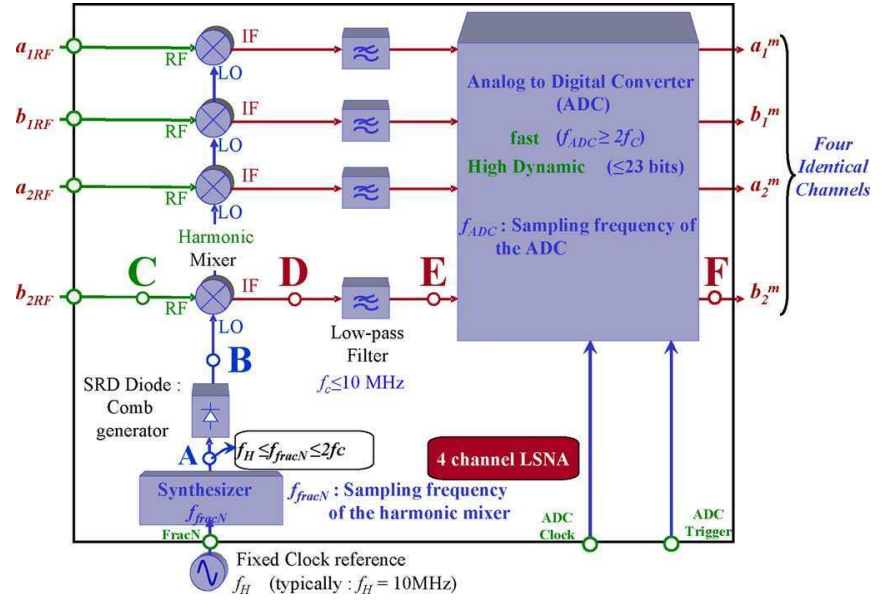

Fig. 1. Sub-sampling principle of the four channel LSNA.

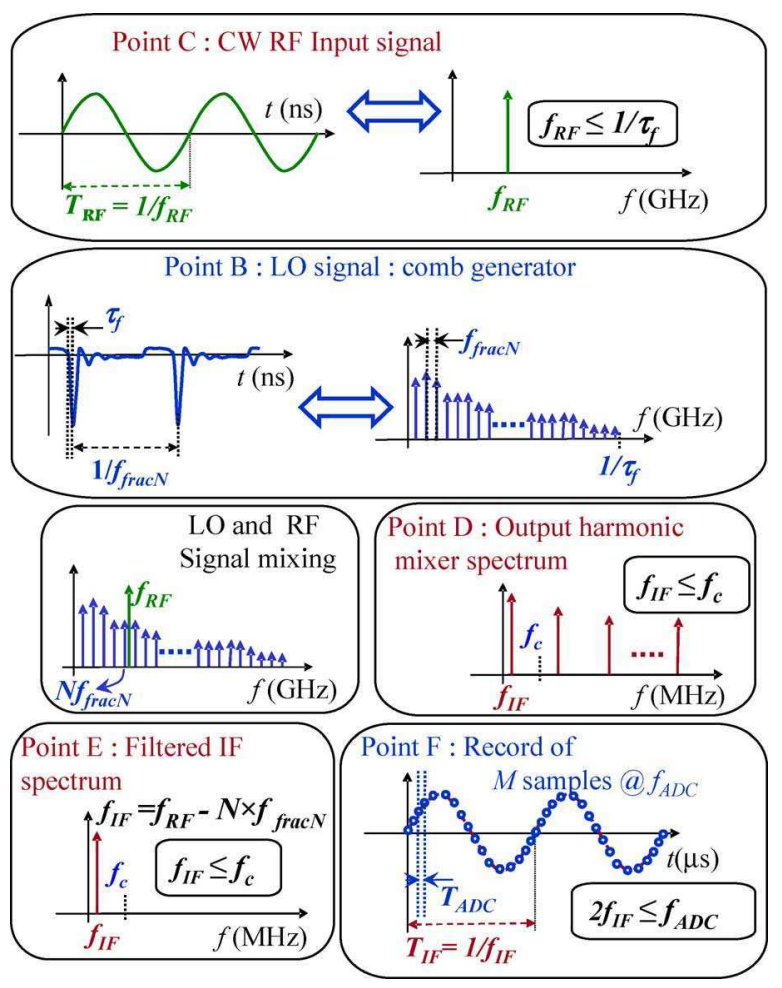

Fig. 2. Representation of the different signals of the LSNA channel.

\section{Wideband Multisine MEASUREMENTS USING THE LSNA}

In this section, the basic measurement principle of the LSNA is briefly reviewed for $\mathrm{CW}$ microwave signal and harmonics measurements. A block diagram that illustrates the harmonic sub-sampling technique performed by the synchronized four channel LSNA is given in Fig. 1.

Fig. 2 gives a frequency and a time-domain representations of signals at Points A-F of Fig. 1 when the RF signal at Point $C$ is a $\mathrm{CW}$ signal at a frequency $f_{\mathrm{RF}}$. A low-pass filter is used to extract the IF image of the RF signal. The cutoff frequency $f_{c}$ of the low-pass filter needs to be lower than $f_{\mathrm{ADC}} / 2$

An ADC is then used to sample the IF signal. $M$ samples of raw data are recorded. A calibration procedure for wideband multisine measurement is required to get error-corrected data. 


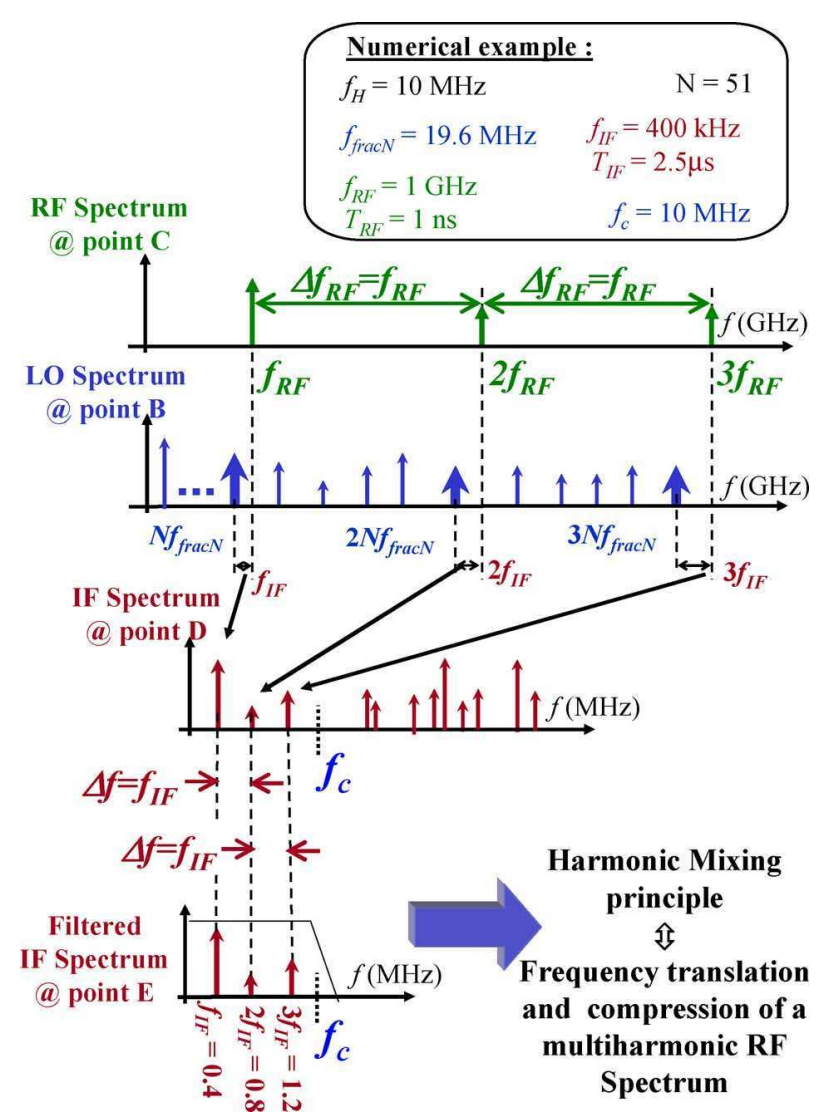

Fig. 3. RF to IF frequency translation and compression principle.

Fig. 3 illustrates the RF to IF frequency translation and compression mechanisms when the input RF signal at Point $\mathrm{C}$ is now composed of the first three harmonics of an RF tone at a frequency $f_{\mathrm{RF}}$.

The second graph of the Fig. 3 represents, at Point B, the spectrum of the strobe signal required for the sub-sampling process. As shown, the spectrum is a comb signal with a frequency spacing $f_{\text {fracN }}\left(f_{\text {frac } N}=19.6 \mathrm{MHz}\right.$ in the numerical example of Fig. 3)

At Point $\mathrm{D}$, a wide spectrum resulting from multiple mixing products between the RF spectral components and the local oscillator (LO) spectral components is obtained.

At Point E, we get a low-pass filtered IF spectrum, which is a low-frequency copy of the RF input spectrum. In the numerical example of Fig. 3, the frequency $f_{\mathrm{IF}}$ of the filtered spectrum is equal to $0.4 \mathrm{MHz}$. The frequency $f_{\mathrm{IF}}$ is the result of the mixing between $f_{\mathrm{RF}}\left(f_{\mathrm{RF}}=1 \mathrm{GHz}\right)$ and $N f_{\text {frac } N}=999.6 \mathrm{MHz}$ with $N=51\left(N \times f_{\text {frac } N}=51 \times 19.6 \mathrm{MHz}\right)$. The frequency $2 f_{\mathrm{IF}}$ of the filtered spectrum is equal to $0.8 \mathrm{MHz}$ and is the result of the mixing between $2 f_{\mathrm{RF}}\left(2 f_{\mathrm{RF}}=2 \mathrm{GHz}\right)$ and $2 N f_{\mathrm{Frac} N}=$ $1.9992 \mathrm{GHz}$ with $N=51\left(2 \times N \times f_{\text {Frac } N}=2 \times 51 \times\right.$ 19.6 MHz).

As depicted in Fig. 1, the filtered IF signal is then digitized using high-dynamic range ADCs ( $50 \mathrm{MHz}$; in our case, 14 bits). The maximum sub-sampling frequency $f_{\text {frac } N}$ of the LSNA is in the order of $25 \mathrm{MHz}$. The IF low-pass filter bandwidth is in the order of $10 \mathrm{MHz}$.

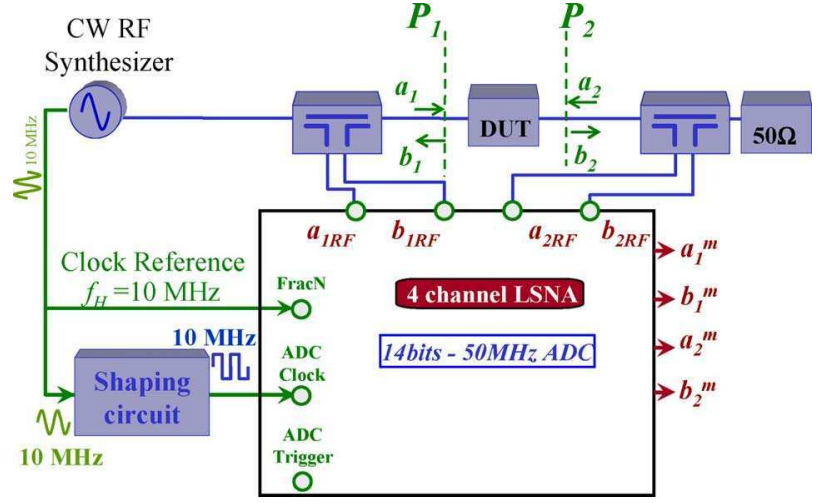

Fig. 4. Block diagram of the LSNA system used for CW measurements.

This instrument can be used to measure periodic microwave signals.

A block diagram of the complete LSNA test bench including two bidirectional couplers is depicted in Fig. 4.

The basic relationships linking the measured data and the error corrected data of a two-port device are described as follows:

$$
\begin{aligned}
\left(\begin{array}{c}
a_{1}^{i} \\
b_{1}^{i} \\
a_{2}^{i} \\
b_{2}^{i}
\end{array}\right)=\left|K^{i}\right| e^{j \cdot \varphi_{K}^{i}} \times\left(\begin{array}{cccc}
1 & \beta_{1}^{i} & 0 & 0 \\
\gamma_{1}^{i} & \delta_{1}^{i} & 0 & 0 \\
0 & 0 & \alpha_{2}^{i} & \beta_{2}^{i} \\
0 & 0 & \gamma_{2}^{i} & \delta_{2}^{i}
\end{array}\right) \\
\\
\\
\end{aligned}
$$

Indices 1 and 2 represent the device port number, while $i$ is the frequency index. The relative error coefficients $\beta_{1}^{i}, \gamma_{1}^{i}$, $\delta_{1}^{i}, \alpha_{2}^{i}, \beta_{2}^{i}, \gamma_{2}^{i}, \delta_{2}^{i}$, and the absolute error terms $\left(\left|K^{i}\right|\right.$ and $\left.\varphi_{K}^{i}\right)$ must be determined by a calibration procedure. As mentioned in Section I, this paper will not focus on the determination of $\beta_{1}^{i}$, $\gamma_{1}^{i}, \delta_{1}^{i}, \alpha_{2}^{i}, \beta_{2}^{i}, \gamma_{2}^{i}, \delta_{2}^{i}$, and $\left|K^{i}\right|$ using well-known SOLT, TRL, or LRRM relative calibrations and absolute power calibration. Instead, this paper focuses the determination of the term $\varphi_{K}^{i}$ that requires a phase-calibration step, which is the most problematic for the time-domain measurements of wideband multisines. The phase calibration consists of determining the phase relationships between the RF components $\left(f_{\mathrm{RF}}, 2 f_{\mathrm{RF}}, 3 f_{\mathrm{RF}}, \ldots, n f_{\mathrm{RF}}\right)$ and the corresponding IF images $\left(f_{\mathrm{IF}}, 2 f_{\mathrm{IF}}, 3 f_{\mathrm{IF}}, \ldots, n f_{\mathrm{IF}}\right)$ shown in Fig. 5.

For that purpose, the phase relationships $\left(\theta_{2}-\theta_{1}\right)$ between $f_{\mathrm{RF}}$ and $2 f_{\mathrm{RF}},\left(\theta_{3}-\theta_{1}\right)$ between $f_{\mathrm{RF}}$ and $3 f_{\mathrm{RF}}$, and $\left(\theta_{n}-\theta_{1}\right)$ between $f_{\mathrm{RF}}$ and the $n$th harmonic of $f_{\mathrm{RF}}$ must be perfectly known. $\theta_{1}$ is an arbitrary phase reference, which can be set to 0 . As a consequence, an HPR generator must be used and connected at plane 2 ( $P_{2}$ in Fig. 4$)$ for the phase calibration of the setup. This is illustrated in Fig. 6. During this phase calibration, the phase relationships $\left(\theta_{2}^{\prime}-\theta_{1}^{\prime}\right),\left(\theta_{3}^{\prime}-\theta_{1}^{\prime}\right)$, and $\left(\theta_{n}^{\prime}-\theta_{1}^{\prime}\right)$ are measured and error correction terms $\left(\theta_{1}^{\prime}-\theta_{1}\right),\left(\theta_{2}^{\prime}-\theta_{2}\right)$, and $\left(\theta_{n}^{\prime}-\theta_{n}\right)$ can be extracted.

The frequency $f_{0}$ of the RF sinusoidal signal driving the HPR generator input can be tuned between $600 \mathrm{MHz}-1.2 \mathrm{GHz}$. Therefore, it generates harmonic 


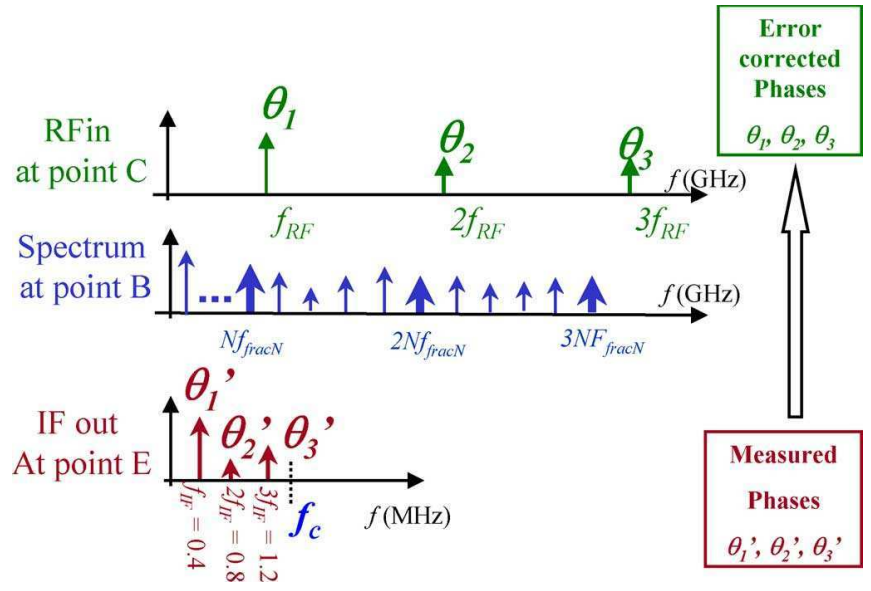

Fig. 5. Phase relationships between RF and IF spectral components.

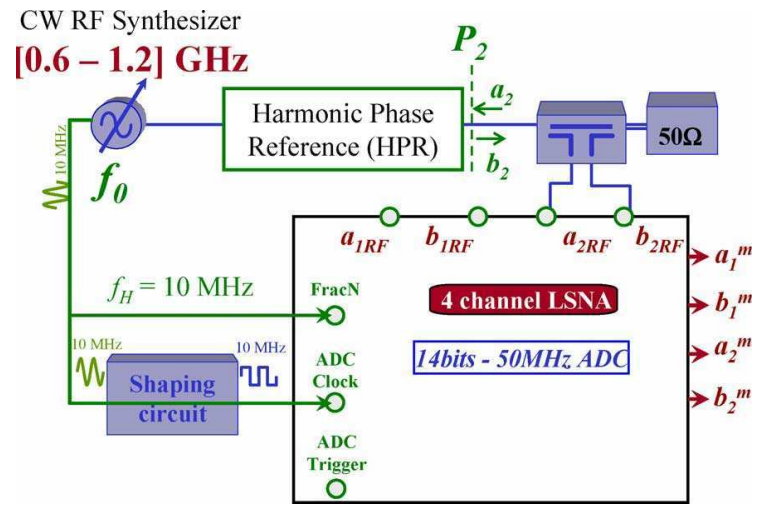

Fig. 6. Phase calibration of the LSNA with the classical HPR.

components at $f_{0}, 2 f_{0}, 3 f_{0}, \ldots, N^{\prime} f_{0} . f_{0}$ is tuned such that $k f_{0}$ is exactly equal to $f_{\mathrm{RF}} . k$ is an integer and $f_{\mathrm{RF}}$ is the frequency of interest. The sampling frequency $f_{\text {frac } N}$ is generally tuned close to $20 \mathrm{MHz}$ and must remain exactly the same during the calibration procedure and the device-under-test measurements. The phase values $\theta_{1}, \theta_{2}$, and $\theta_{3}$ at frequencies $f_{0}, 2 f_{0}$, and $3 f_{0}$, generated by the classical HPR generator, are perfectly known. Measuring $\theta_{1}^{\prime}, \theta_{2}^{\prime}$, and $\theta_{3}^{\prime}$ during the phase-calibration procedure enables the determination of the value of $\varphi_{K}^{i}$ expressed in (1) and depicted in Fig. 5. The values of $\varphi_{K}^{i}$ are deduced from the following:

$$
\begin{cases}\varphi_{K}^{1}=\theta_{1}^{\prime}-\theta_{1}, & \text { for }=1 \Leftrightarrow f=f_{0} \\ \varphi_{K}^{2}=\theta_{2}^{\prime}-\theta_{2}, & \text { for } i=2 \Leftrightarrow f=2 f_{0} \\ \varphi_{K}^{3}=\theta_{3}^{\prime}-\theta_{3}, & \text { for } i=3 \Leftrightarrow f=3 f_{0} .\end{cases}
$$

When the above sub-sampling technique is applied to the measurement of modulated microwave signals, having modulation bandwidths higher than $20 \mathrm{MHz}$, things become much more complicated. Effectively, in that case, descrambling techniques of the measured IF spectral components are necessary after downconversion.

In the following, a periodic modulated signal is represented by a multisine having a bandwidth larger than $20 \mathrm{MHz}$. If such a

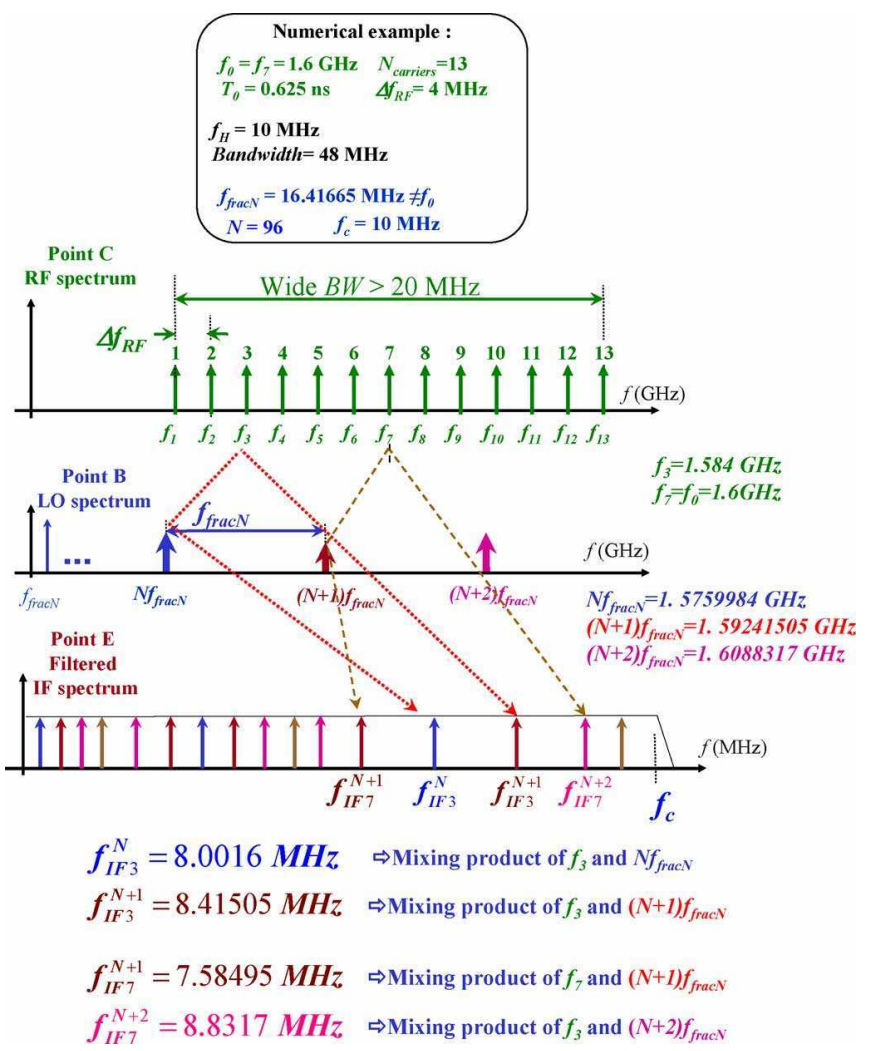

Fig. 7. Illustration of mixing principle for wideband multisine RF signal.

signal is applied to the RF input (Point C) depicted in Fig. 1, the RF to IF conversion process performed by the LSNA is shown in Fig. 7. Only spectral components falling in the 10-MHz bandwidth of the IF filter are measured by the ADC.

In Fig. 7, the IF spectrum is the result of multiple $f_{\mathrm{IFM}}^{N}$ mixing products of the $M$ th RF frequency $\left(f_{M}\right)$ and the $N$ th harmonic of $f_{\text {frac } N}$. As an example, dotted lines in Fig. 7 illustrate double mixing of $f_{3}$ and $f_{7}$ with two successive harmonics of $f_{\text {frac } N}$.

Thus, the IF spectrum does not directly provide a low-frequency copy of the RF signal. Since the IF spectral components are scrambled, a descrambling technique is then required, as explained in [19].

However, the main problem lies in the phase-calibration procedure of the system for such wideband signal measurements. We proposed a method of performing a phase calibration using a narrowband multisine reference generator to perform wideband measurements of interleaved IF signals [20].

This method is complex and it suffers from propagation of errors such that the uncertainty of the phase increases when moving toward both edges of the modulation frequency range. A solution has been presented in [23] to address this problem using a pulsed modulation of the $\operatorname{Frac} N$ output signal to capture samples of the RF signal at very low frequencies. To avoid adding another modulation module in the LSNA, in this paper we propose another solution using an appropriate multisine reference generator for the calibration of the setup. This reference generator is a multisine having a fine frequency grid, which is $20 \mathrm{MHz}$ for our work reported here. This is now presented in Section III. 


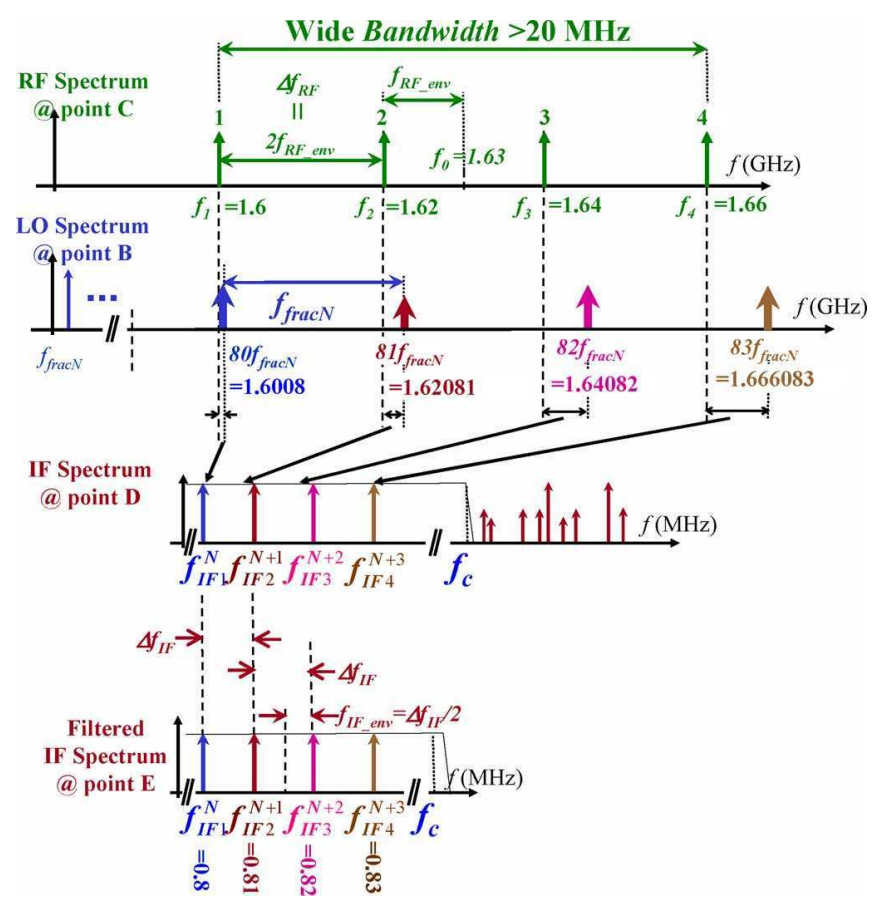

Fig. 8. Frequency translation and compression principle with a multisine having a modulation bandwidth higher than twice the IF bandwidth $(\geq 20 \mathrm{MHz}$ ).

\section{PResentation of the Proposed Method}

\section{A. Description of the Principle}

The key idea is, in fact, quite simple. It uses the LSNA for multisine measurements in the same manner as it is used for $\mathrm{CW}$ measurements. For $\mathrm{CW}$ measurements, we consider the carrier frequency and its harmonics $\left(f_{\mathrm{RF}}, 2 f_{\mathrm{RF}}, 3 f_{\mathrm{RF}}, \ldots, N f_{\mathrm{RF}}\right)$ during both LSNA calibration and device-under-test measurement. It means that a phase reference generator providing exactly $f_{\mathrm{RF}}$ and its harmonics is required for the phase-calibration step. The microwave source at $f_{\mathrm{RF}}$ and the synthesizer providing the strobe signal for harmonic sub-sampling are synchronized using a common $10-\mathrm{MHz}$ reference signal. The sampling frequency $f_{\text {frac } N}$ is carefully chosen such that one of its harmonics $N f_{\text {frac } N}$ is very close to $f_{\mathrm{RF}}$ [2]. Therefore, a well-conditioned frequency compression mechanism is achieved. Once the sub-sampling frequency $f_{\text {frac } N}$ is carefully tuned, it remains constant during both phase calibration and measurement.

The same process is used for multisine measurements having, for example, a $20-\mathrm{MHz}$ frequency grid.

This is described in Fig. 8, where the $60-\mathrm{MHz}$ bandwidth RF signal consists of four tones with $20-\mathrm{MHz}$ frequency spacing. It has a $1.63-\mathrm{GHz}$ center frequency and a $10-\mathrm{MHz}$ envelope frequency. The filtered IF image signal also consists of four tones. It has a $0.815-\mathrm{MHz}$ center frequency and a $5-\mathrm{kHz}$ envelope frequency. The frac $N$ frequency is tuned to $20.01 \mathrm{MHz}$ for this configuration.

In such conditions, the phase calibration is performed using a new HPR generator providing harmonics of a $20-\mathrm{MHz}$ sinewave, which corresponds to the tone spacing of the microwave multisine that we want to characterize. We have built this new phase reference generator combining a $20-\mathrm{MHz}$ synthesizer, an amplifier, a step recovery diode, and a differentiator, as described in Section III-B.

It is very important to note here that the ADCs are triggered by a transistor-transistor logic (TTL) signal. The trigger frequency $f_{\text {trig }}$ is linked to the fast Fourier transform grid $\left(f_{\mathrm{FFT} \text {-grid }}\right)$ used for spectral calculation

$$
f_{\text {trig }}=p \times f_{\mathrm{FFT}_{\text {_grid }}}(p: \text { integer })
$$

with

$$
f_{\mathrm{FFT}_{\text {_grid }}}=\frac{f_{\mathrm{ADC}}}{N_{\mathrm{ADC}}}
$$

where $f_{\mathrm{ADC}}$ and $N_{\mathrm{ADC}}$ are, respectively, the sampling frequency of the ADCs (different from the sub-sampling frequency $\left.f_{\text {frac } N}\right)$ and the number of recorded samples of the ADCs. One must also make sure that the period of the trigger signal equals the IF envelope period $T_{\mathrm{IF} \text { _env }}$ or an integer multiple of this period

$$
T_{\text {trig }}=M \times T_{\mathrm{IF}_{\text {env }}}(M: \text { integer })
$$

with

$$
f_{\mathrm{IF} \_ \text {env }}=q \times f_{\mathrm{FFT}_{\text {_grid }}}(q: \text { integer }) \text {. }
$$

From (3), (5), and (6), $M$ can be defined as

$$
M=\frac{p}{q}
$$

This trigger signal is synthesized with an arbitrary waveform generator phase locked with the $10-\mathrm{MHz}$ reference generated by the multitone RF generator.

Both phase and measurement configurations of the setup are, respectively, shown in Figs. 9 and 10.

In the numerical example of Fig. 9, the sampling frequency $f_{\mathrm{ADC}}$ of the ADC is set to $25 \mathrm{MHz}$. The number of samples $N_{\mathrm{ADC}}$ is set to 25000 . This results in a $1-\mathrm{kHz}$ frequency resolution.

The IF spectrum, which is an image of the RF spectrum, has four tones, as described in Fig. 8 at $f_{\mathrm{IF} 1}^{N}=800 \mathrm{kHz}$, $f_{\mathrm{IF} 2}^{N+1}=810 \mathrm{kHz}, f_{\mathrm{IF} 3}^{N+2}=820 \mathrm{kHz}$, and $f_{\mathrm{IF} 4}^{N+3}=830 \mathrm{kHz}$ $\left(\Delta f_{\mathrm{IF}}=10 \mathrm{kHz}\right)$. These four IF tones are the result of the harmonic mixing process realized, respectively, with the successive 80th, 81st, 82nd, and 83rd harmonics of $f_{\text {frac } N}$. The frequency $f_{\text {frac } N}$ is set to $20.01 \mathrm{MHz}$ in that case. The envelope period of the IF signal is equal to $200 \mu \mathrm{s}\left(T_{\mathrm{IF} \_ \text {env }}=200 \mu \mathrm{s} \Leftrightarrow f_{\mathrm{IF} \_ \text {env }}=\right.$ $5 \mathrm{kHz}$ ). Choosing $p$ equal to 5 and $M$ equal to 1 implies the use of a $200-\mu$ s trigger signal $\left(T_{\text {trig }}=200 \mu \mathrm{s} \Leftrightarrow f_{\text {trig }}=5 \mathrm{kHz}\right)$.

Fig. 11 shows a time-domain representation of the signal at the ADC output and a time-domain representation of the associated trigger signal.

The trigger signal ensures that the acquisition starting time is always the same in regard to the envelope of the signal (with the limitation of the phase jitter of the trigger signal). This is a key point of this measurement system that this trigger signal remains the same during the calibration and measurement steps. 


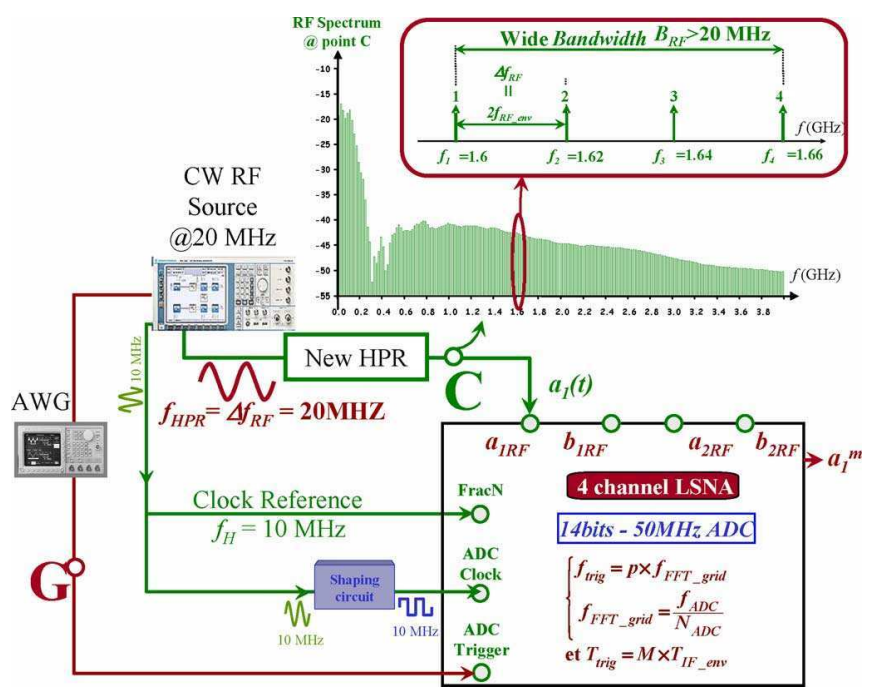

Fig. 9. Configuration of the setup for the phase-calibration step.

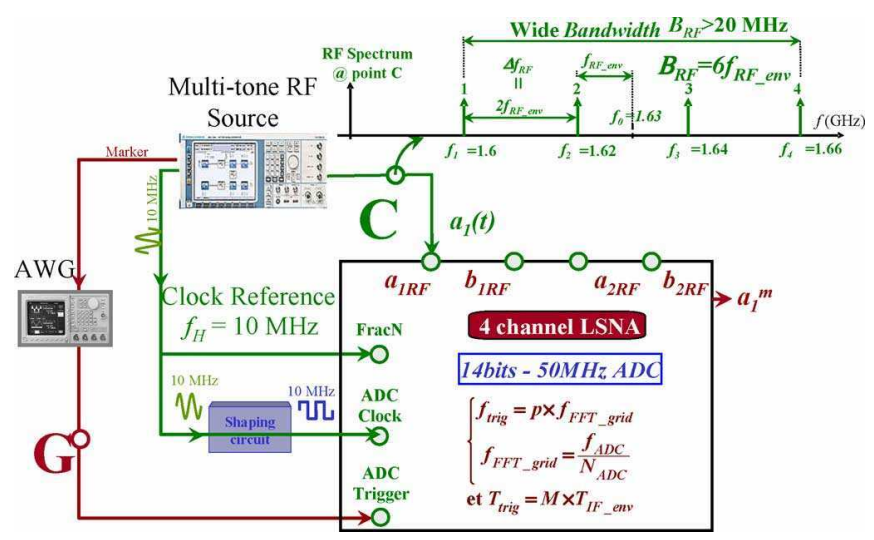

Fig. 10. Configuration of the setup for device measurement .

The ADC performs an over-sampling of the IF two-tone signal and outputs 25000 voltage points sampled every $40 \mathrm{~ns}$, as shown in the zoom time-domain representation of Fig. 11.

Using this technique, no scrambling occurs in the IF spectrum. The LSNA proceeds to an RF to IF multisine frequency translation and compression in the same manner as for a $\mathrm{CW}$ carrier and its harmonics.

\section{B. Description of the Phase Reference Generator}

The new HPR generator that has been built using parts of an microwave transition analyzer is described in Fig. 12.

This new HPR is based on the use of a step recovery diode driven by a low-frequency sinusoidal signal, of which frequency $f_{\mathrm{HPR}}$ is equal to the $\mathrm{RF}$ tone spacing $\left(\Delta f_{\mathrm{RF}}\right)$ of the multisine used for measurements. In our case, $f_{\mathrm{HPR}}$ is $20 \mathrm{MHz}$. A nonlinear transmission line is added at the output of the step recovery diode to reduce the duration of the pulse and, in particular, the falling time of the pulse such that the output RF frequency bandwidth increases. The new HPR generator includes a 16-dB attenuator to obtain the appropriate desired peak voltage to protect the sampling gate when performing the phase calibration of the LSNA.

The output spectrum of this HPR generator is a comb signal and it has the same type of frequency response as the one used to feed the sampling head of the LSNA (LO port

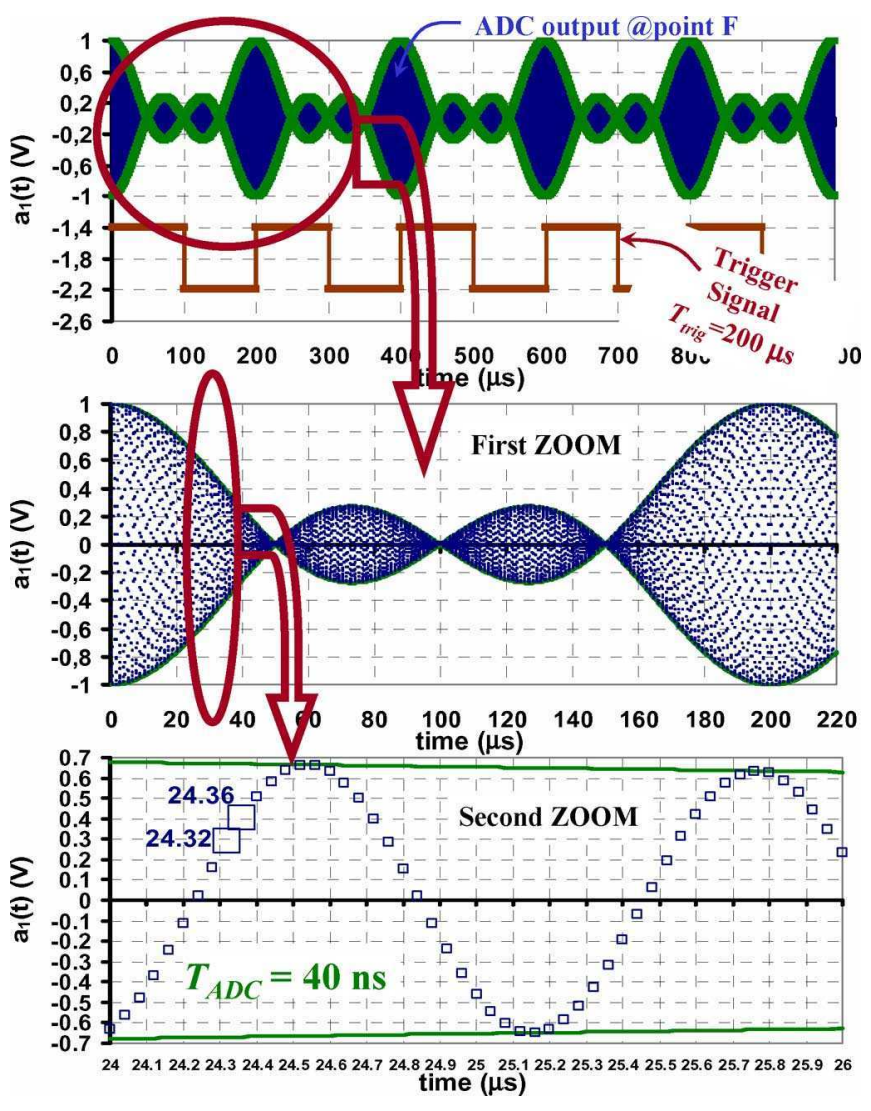

Fig. 11. Time-domain representation of the ADC output signal and the associated trigger signal for a wideband two-tone acquisition.

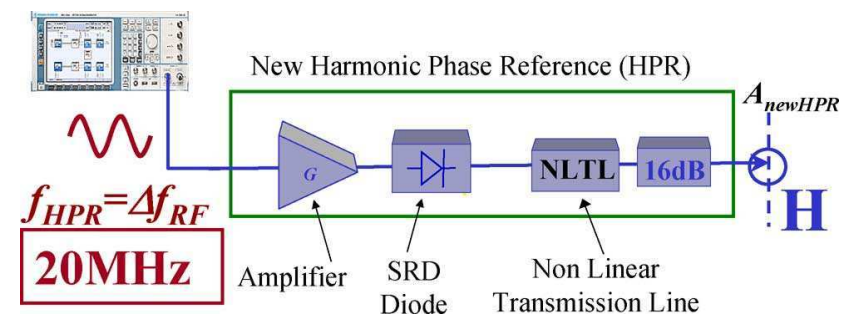

Fig. 12. Description of the new HPR.

at point $\mathrm{B}$ in Fig. 1). In the frequency domain, the output of the new HPR is represented by harmonic components at $f_{\mathrm{HPR}}, 2 f_{\mathrm{HPR}}, 3 f_{\mathrm{HPR}}, \ldots, M f_{\mathrm{HPR}}$.

\section{Calibration Procedure of the Phase Reference Generator}

The HPR has been calibrated on a dense 20-MHz frequency grid up to $4 \mathrm{GHz}$ by NMDG using a 50-GHz sampling oscilloscope. The measurement setup is described in Fig. 13.

It should be noticed that normally the $20-\mathrm{MHz}$ excitation signal is used both as an input signal of the new HPR and as a trigger signal of the equivalent-time oscilloscope using a power splitter. However, to use exactly the same setup as during the phase calibration of the LSNA (which does not require a trigger signal, but a common $10-\mathrm{MHz}$ reference signal), the setup is adapted such that the amplified 10-MHz reference signal of the $\mathrm{CW}$ synthesizer is used to trigger the sampling oscilloscope.

A 10-dB attenuator is added to the new HPR to avoid any nonlinearities introduced by the $50-\mathrm{GHz}$ sampling oscilloscope. 


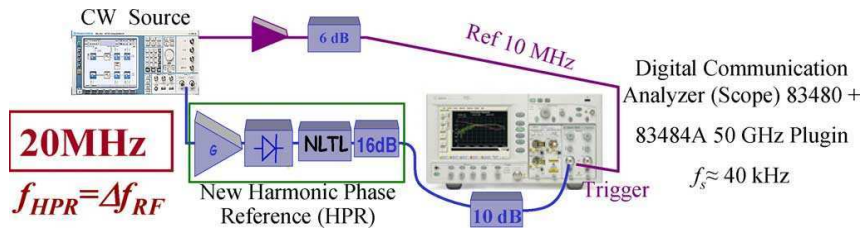

Fig. 13. Measurement setup used during calibration of the new HPR.
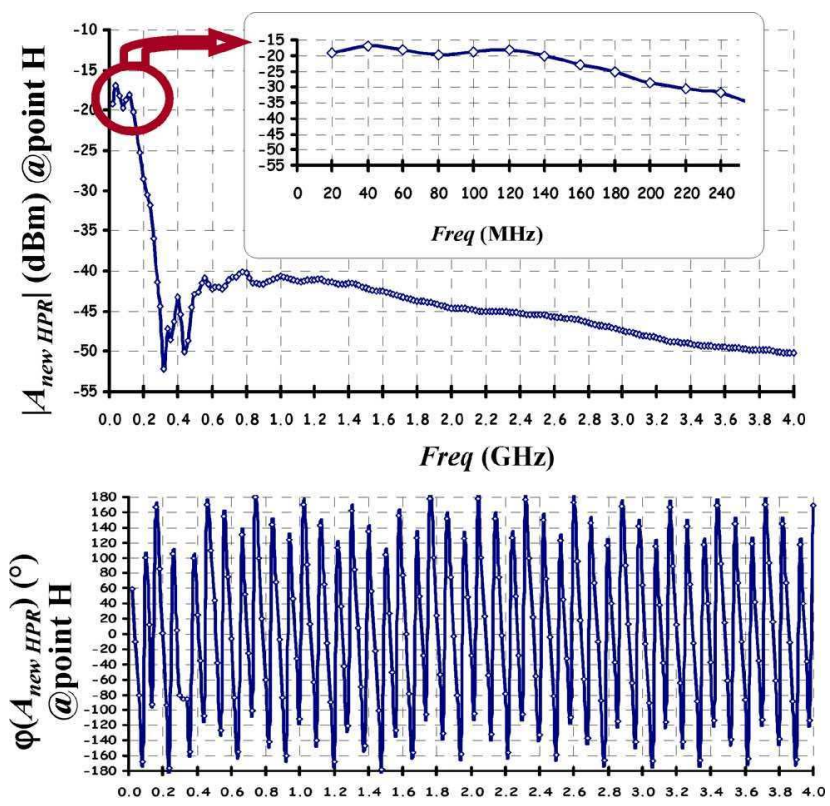

Freq $(\mathbf{G H z})$

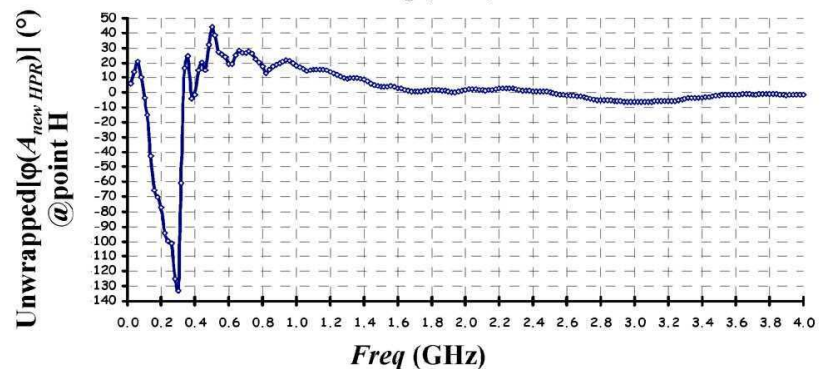

Fig. 14. Frequency-domain representation of the output signal provided by the new HPR.

Afterwards, the 10-dB attenuator is deembedded based on its two-port $S$-parameters.

As the maximum frequency of the HPR is $4 \mathrm{GHz}$, magnitude and phase distortion of the 50-GHz sampling oscilloscope can be reasonably neglected [3], [4]. However, one must deal with errors due to the time base distortion and time base drift of the sampling oscilloscope. The offset and gain error of the sampling oscilloscope is eliminated by performing the built-in plug-in calibration up front, while the jitter is neglected. Indeed, assuming a symmetrical probability density function of the jitter, it is known that the jitter does not introduce any phase distortion [23]. However, it has a low-pass effect on the amplitude. Since only the phase information is used, this effect is not compensated for and, as such, the amplitude characteristic shown in Fig. 14 includes the low-pass effect of the jitter.

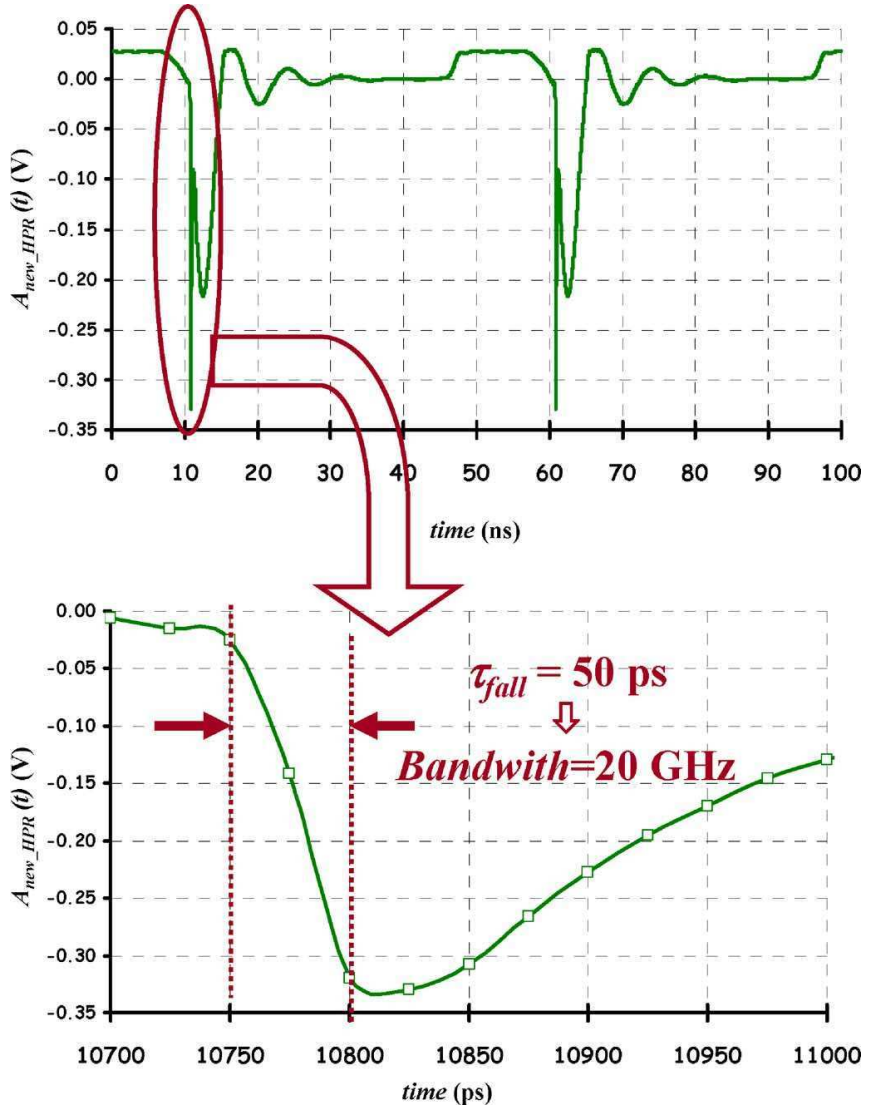

Fig. 15. Time-domain representation of the output signal provided by the new HPR.

The time base distortion is estimated [25] using a 100-ns time window, corresponding to two periods of the 20-MHz excitation signal.

In our experience, the known time base discontinuities are equal to $4 \mathrm{~ns}$. Reference [26] is used to compensate for this distortion as part of the measurement of the pulse. Using two periods, one can verify if this correction is appropriate because one out of two spectral lines should be at the noise level after time base compensation.

Using the amplified 10-MHz reference signal of the synthesizer instead of splitting the $20-\mathrm{MHz}$ excitation signal, it was found that it is essential to estimate and compensate the time base drift before averaging the measured pulse. Averaging is required to reduce the uncertainty on the measured phase of each spectral component.

Fig. 15 shows that the fall time of the generated pulse equals $50 \mathrm{ps}$ and indicates that spectral components are present up to $20 \mathrm{GHz}$.

As a sanity check, the obtained phase of each spectral component is compared to the one obtained when the $20-\mathrm{MHz}$ excitation is also used as a trigger signal, resulting in a less pronounced time base drift.

Finally, although being very small for the considered frequency range, the mismatch of both the HPR generator and the sampling oscilloscope is taken into account. At the same time, the 10-dB attenuator used to avoid nonlinearities of the sampling oscilloscope itself is deembedded. As the final result of the calibration, two files are provided. One file contains the phase in- 


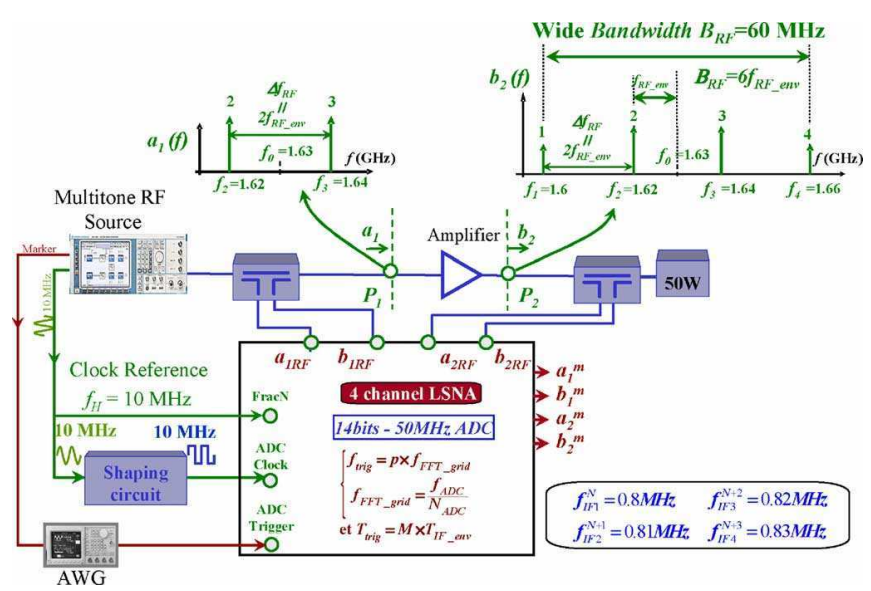

Fig. 16. Principle of phase measurements of power amplifier intermodulations.

formation of the HPR generator on a $20-\mathrm{MHz}$ grid up to $4 \mathrm{GHz}$ when terminated in $50 \Omega$. The other contains the mismatch information of the HPR generator obtained using a vector network analyzer. Both files are required during the phase calibration of the LSNA.

This HPR generator is used during the phase-calibration step of the LSNA, configured as described in Section III-A. The principle of the phase-calibration step is exactly the same as the one previously described in Section II. The phase relationships between all the frequency components $\left(f_{\mathrm{HPR}}, 2 f_{\mathrm{HPR}}, 3 f_{\mathrm{HPR}}, \ldots, m f_{\mathrm{HPR}}\right)$ at the output of the new HPR generator are known based on the above calibration procedure of the HPR generator itself.

\section{MEASUREMENT RESUlts}

Two types of measurements will be examined for the two following applications:

- two-tone measurements for the intermodulation characterization of the power amplifier;

- three- and five-tone measurements to validate a possible future use for the multipactor characterization in multicarrier satellite payloads.

\section{A. Phase Measurements of Power Amplifier Intermodulation Products}

A $2-\mathrm{W}$ output power $30-\mathrm{dB}$ gain $50-\Omega$ power amplifier at $1.6 \mathrm{GHz}$ has been measured. The amplifier is excited by a twotone signal generated by a Rohde \& Schwarz SMU200A source, as sketched in Fig. 16. The tone spacing is equal to $\Delta f_{\mathrm{RF}}=$ $20 \mathrm{MHz}$

The calibration procedure has been performed at four different frequencies $f_{1}=1.6 \mathrm{GHz}, f_{2}=1.62 \mathrm{GHz}, f_{3}=$ $1.64 \mathrm{GHz}$, and $f_{4}=1.66 \mathrm{GHz}$ corresponding to a $60-\mathrm{MHz}$ total RF bandwidth.

Table I indicates the parameter settings for this two-tone characterization.

The configuration of the test bench is the same as the one used for the calibration procedure. Harmonics of carriers are not considered in this study.

The LSNA input range is fixed for all input powers, resulting in a dynamic range of $52 \mathrm{~dB}$. If desired, this dynamic range
TABLE I

PARAMETER SETTINGS FOR TwO-TONE CHARACTERIZATION

\begin{tabular}{ccc}
\hline \hline Parameter & Value & Unit \\
\hline$f_{0}$ & 1.63 & $\mathrm{GHz}$ \\
$B_{R F}$ & 60 & $\mathrm{MHz}$ \\
$\Delta f_{R F}$ & 20 & $\mathrm{MHz}$ \\
$f_{R F \_n v}$ & 10 & $\mathrm{MHz}$ \\
$f_{\text {fracN }}$ & 20.01 & $\mathrm{MHz}$ \\
$N$ & 80 & - \\
$f_{A D C}$ & 25 & $\mathrm{MHz}$ \\
$N_{A D C}$ & 25000 & - \\
$T_{\text {record }}$ & 1 & $\mathrm{~ms}$ \\
$f_{F F T \text { grid }}$ & 1 & $\mathrm{kHz}$ \\
$p$ & 5 & - \\
$M$ & 1 & - \\
$f_{\text {trig }}$ & 5 & $\mathrm{kHz}$ \\
$f_{\text {OIF }}$ & 815 & $\mathrm{kHz}$ \\
$N_{\text {carriers }}$ & 4 & - \\
$\Delta f_{I F}$ & 10 & $\mathrm{kHz}$ \\
$f_{I F \text { env }}$ & 5 & $\mathrm{kHz}$ \\
\hline \hline
\end{tabular}

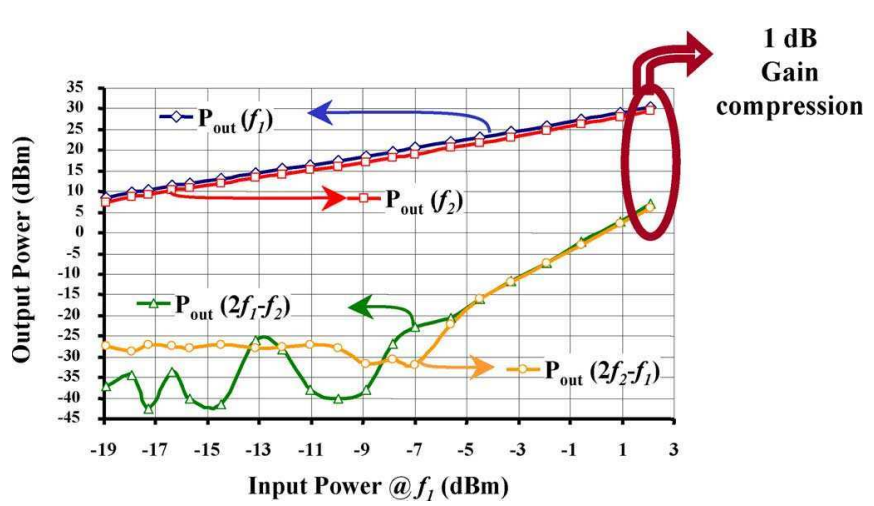

Fig. 17. Measured power characteristics of the amplifier under test.

can be improved by increasing the number of acquisition points $N_{\text {ADC. }}$

Fig. 17 shows the measured power characteristics of the amplifier under test driven by a two-tone signal.

Fig. 18 represents the incident wave $a_{1}$ at $f_{1}=1.62 \mathrm{GHz}$, $f_{2}=1.64 \mathrm{GHz}$ versus input power at $f_{1}=1.62 \mathrm{GHz}$.

Fig. 19 represents the transmitted wave $b_{2}$ at the amplifier output at $f_{1}=1.62 \mathrm{GHz}$ and $f_{2}=1.64 \mathrm{GHz}$ versus input power.

Fig. 20 represents the variation of the magnitude and phase of the output voltage waveforms $b_{2}$ at $2 f_{1}-f_{2}=1.6 \mathrm{GHz}$ and $2 f_{2}-f_{1}=1.66 \mathrm{GHz}$ versus input power at $f_{1}=1.62 \mathrm{GHz}$.

Due to the noise level, the phase is irrelevant for input powers at $f_{1}$ smaller than $-6 \mathrm{dBm}$. The magnitude and phase information of $b_{2}$ at $2 f_{1}-f_{2}=1.6 \mathrm{GHz}$ and $2 f_{2}-f_{1}=1.66 \mathrm{GHz}$ can be very useful for very linear power amplifier design [24] and for the behavioral modeling of nonlinear devices with memory [25].

\section{B. Phase Measurements of Multisines}

Fig. 21 depicts the configuration of the measurement system to perform the phase measurements of a multisine, which consists of five tones with a $20-\mathrm{MHz}\left(\Delta f_{\mathrm{RF}}\right)$ tone spacing. The center frequency is $1.6 \mathrm{GHz}$. The resulting signal has a bandwidth of $80 \mathrm{MHz}$ and is generated using the Rohde \& Schwarz 

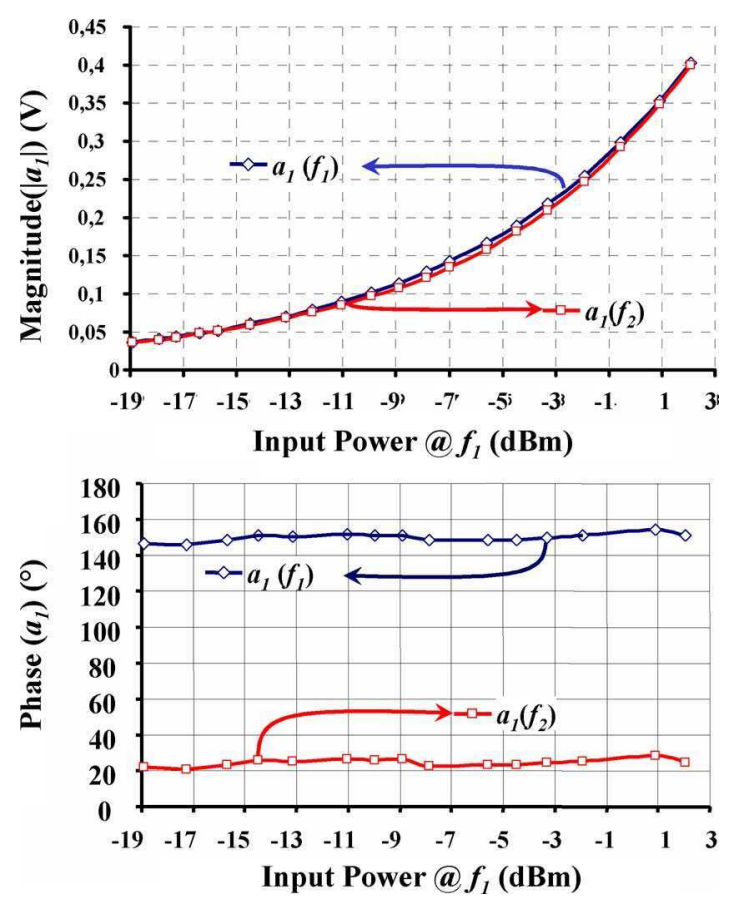

Fig. 18. Incident wave $a_{1}$ at the amplifier input versus input power at $f_{1}$.
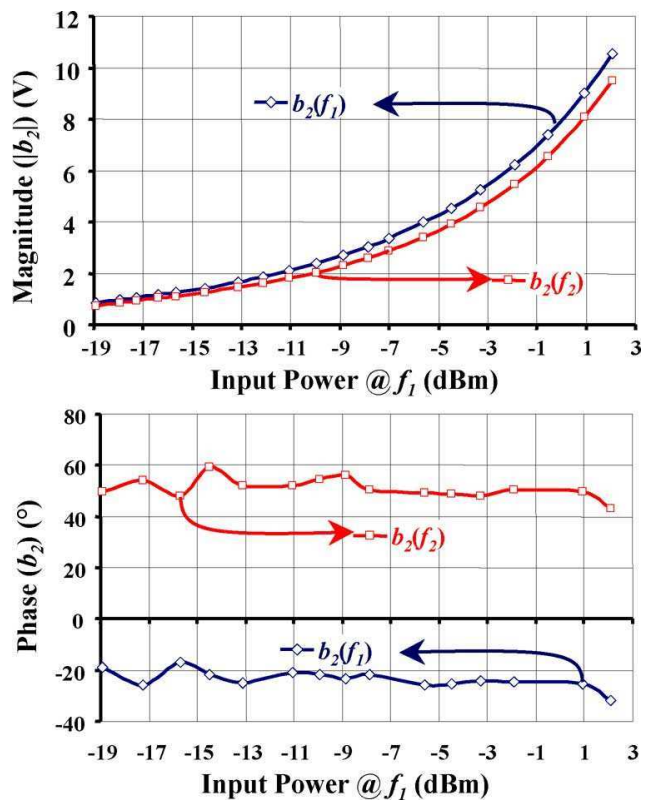

Fig. 19. Transmitted wave $b_{2}$ at the amplifier output (at $f_{1}$ and $f_{2}$ ) versus input power.

source SMU200A. The magnitude of each tone is set to $1 \mathrm{~V}$ and the phase is set to $0^{\circ}$.

Table II indicates the modified parameter settings compared in Table I for the five-tone multisine measurement.

Fig. 22 shows LSNA measurement results of the signal $b_{2}$ present at plane $P_{2}$.

A $0.7-V$ peak voltage and a 7.2-ns pulsewidth are measured. It corresponds to a $13-\mathrm{dBm}$ average power of the multitone $\mathrm{RF}$ source.

The linear phase variation observed in Fig. 22 versus frequency is due to the time delay introduced by the RF cable and
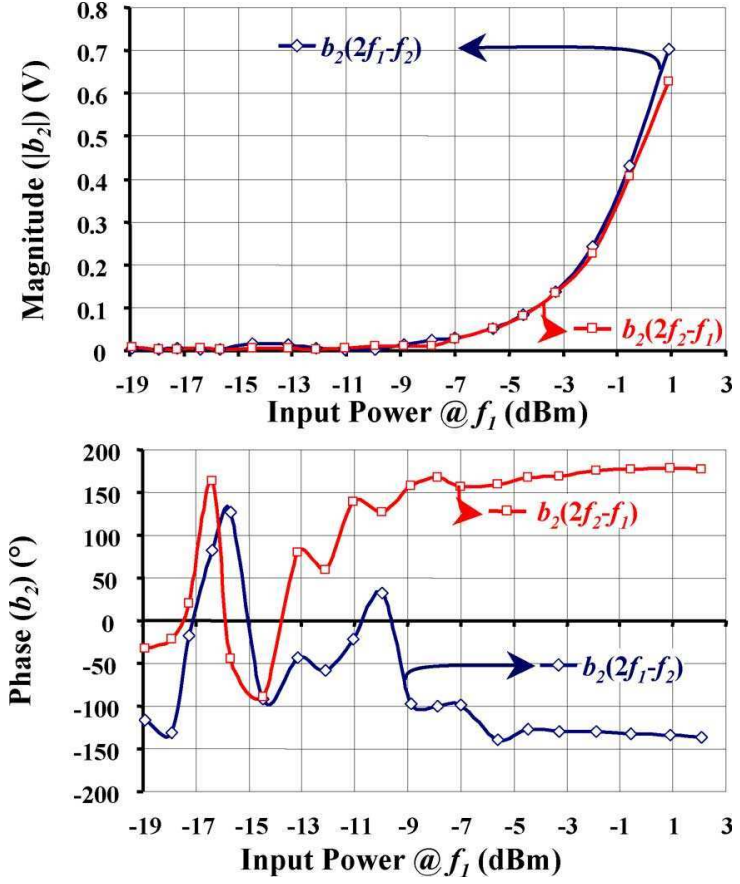

Fig. 20. Transmitted wave $b_{2}$ at the amplifier output (at $2 f_{1}-f_{2}$ and $2 f_{2}-f_{1}$ ) versus input power.

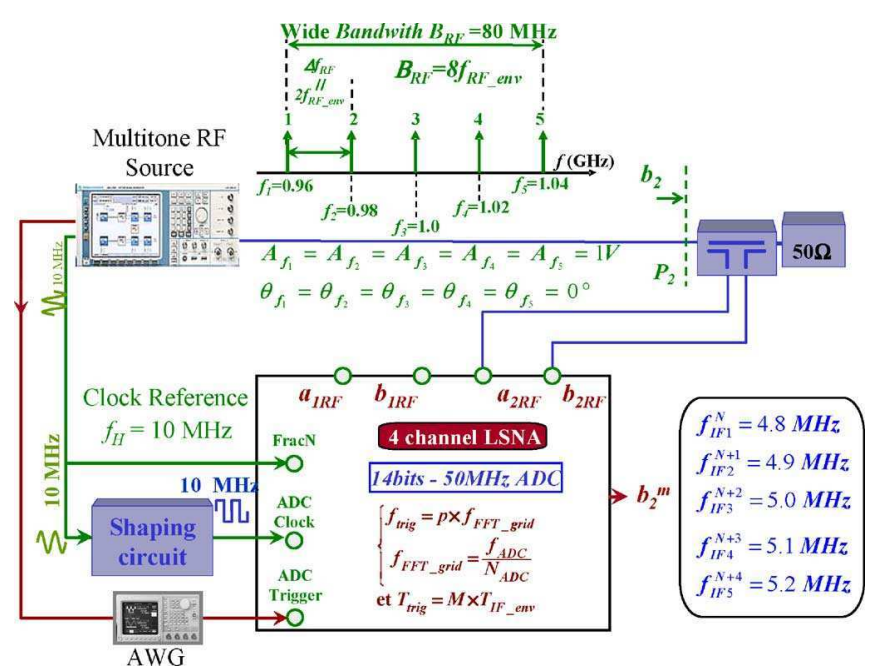

Fig. 21. System configuration for phase measurements of a five-tone multisine.

TABLE II

MOdified PARAmEter SETtings FOR Five-Tone Multisine MEASUREMENT

\begin{tabular}{ccc}
\hline \hline Parameter & Value & Unit \\
\hline$f_{0}$ & 1 & $\mathrm{GHz}$ \\
$B_{R F}$ & 80 & $\mathrm{MHz}$ \\
$f_{\text {frac }}$ & 19.9 & $\mathrm{MHz}$ \\
$N$ & 48 & - \\
$f_{\text {OIF }}$ & 5 & $\mathrm{MHz}$ \\
$N_{\text {carriers }}$ & 5 & - \\
$\Delta f_{I F}$ & 100 & $\mathrm{kHz}$ \\
$f_{I F_{-} \text {env }}$ & 50 & $\mathrm{kHz}$ \\
$M$ & 10 & - \\
\hline
\end{tabular}

connections between the SMU200A source and the reference plane $P_{2}$. 

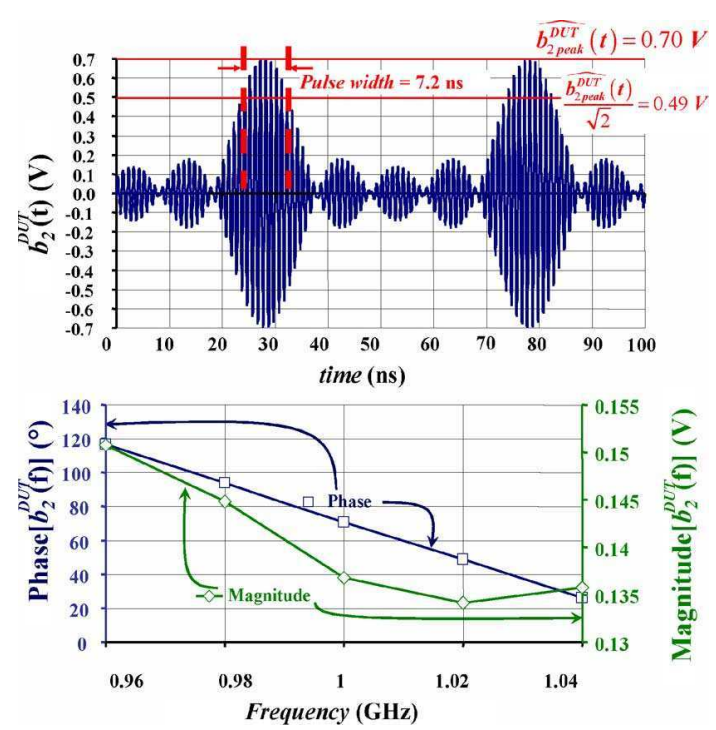

Fig. 22. LSNA measurements of a multisine composed of five tones with a $20-\mathrm{MHz}$ tone spacing.

TABLE III

MODIFIED PARAMETER SETTINGS FOR THREE-TONE MULTISINE MEASUREMENT

\begin{tabular}{ccc}
\hline \hline Parameter & Value & Unit \\
\hline$\Delta f_{R F}$ & 40 & $\mathrm{MHz}$ \\
$f_{R F_{\text {env }}}$ & 20 & $\mathrm{MHz}$ \\
$N_{\text {carriers }}$ & 3 & - \\
\hline
\end{tabular}
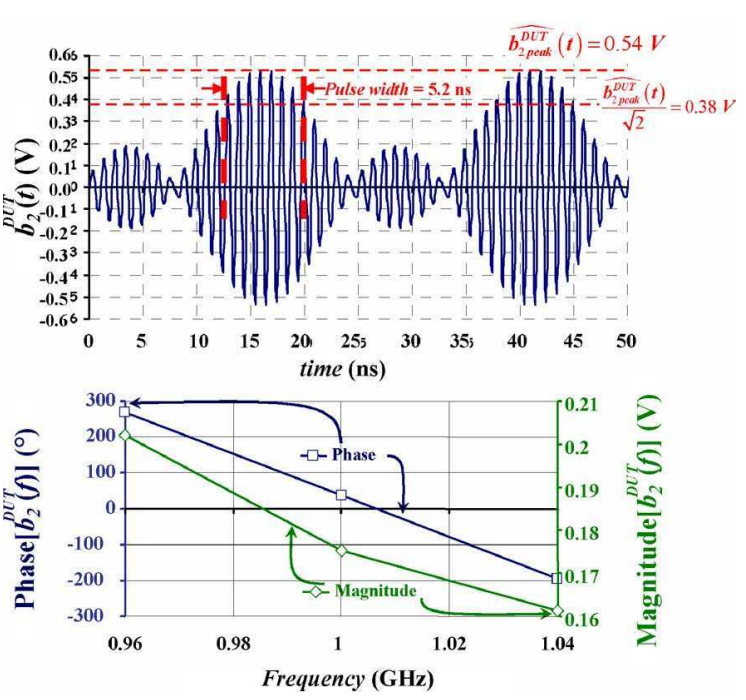

Fig. 23. LSNA measurements of a multisine composed of three tones with a $40-\mathrm{MHz}$ tone spacing.

Using the same setup as the one depicted in Fig. 21, a threetone multisine has been measured. The tone spacing is then set to $40 \mathrm{MHz}\left(\Delta f_{\mathrm{RF}}=40 \mathrm{MHz}\right)$. The magnitude of each tone is set to $1 \mathrm{~V}$ and the phase is set to $0^{\circ}$. The configuration of the LSNA and its calibration remains the same as for the measurement of the five-tone signal. Table III presents the modified parameter settings compared in Table II for the three-tone multisine measurement.

Measurement results of $b_{2}$ at plane $P_{2}$ are given in Fig. 23.

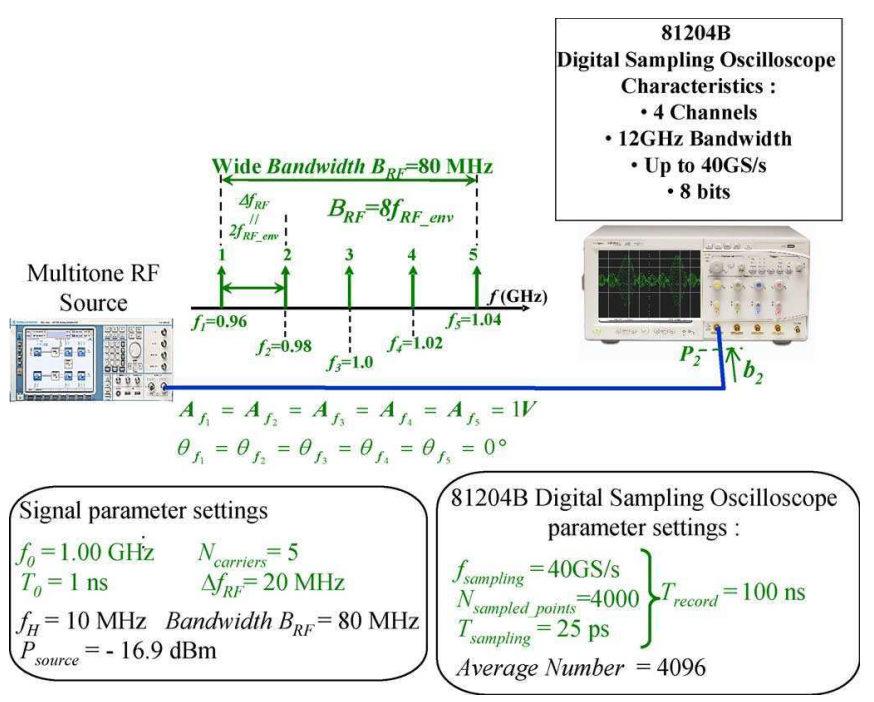

Fig. 24. System configuration for phase measurements of a five-tone multisine with the digital sampling oscilloscope.

The maximum voltage is now equal to $0.54 \mathrm{~V}$, while the pulsewidth decreases to $5.2 \mathrm{~ns}$ for a given $13-\mathrm{dBm}$ average output power of the multitone RF source. The linear phase variation versus frequency (Fig. 23) is also introduced by constant group delay of RF cables and connections between the SMU200A source and the reference plane $P_{2}$

It is very important to show the possibility to measure multisines with 40-MHz spacing, as it corresponds to real conditions in satellite payloads. It is also important to point the potential application to the characterization and the modeling of nonlinear devices.

\section{Comparison Between LSNA and Digital Sampling Oscilloscope Measurements of Wideband Multisines}

To compare the proposed approach described in this paper with digital sampling oscilloscope techniques, we have performed measurements of the five-tone signal previously characterized with a 40-GS/s-12-GHz bandwidth-8-bit digital sampling oscilloscope (Agilent Infinium 81204B), as depicted in Fig. 24.

Fig. 25 shows the digital sampling oscilloscope measurements of the five-tone multisine (80-MHz bandwidth) at the reference plane $P_{2}$.

A $0.7-\mathrm{V}$ peak voltage and a 7.9-ns pulsewidth are measured. It corresponds to an output power of the multitone RF source of $-16.2 \mathrm{dBm}$.

The linear phase variation observed in Fig. 25 versus the frequency is due to the time delay introduced by the RF cable and connections between the SMU200A source and the reference plane $P_{2}$.

Fig. 26 gives a comparison between the LSNA and digital sampling oscilloscope.

A maximum magnitude difference of $0.08 \mathrm{~V}$ can be observed.

A constant phase offset (in the order of $60^{\circ}$ ) is observed between the two measurements. It corresponds to different phase settings of the internal LO of the signal generator for two different experiments and measurements. The signal generator (SMU200A source) has been switched off and on between 

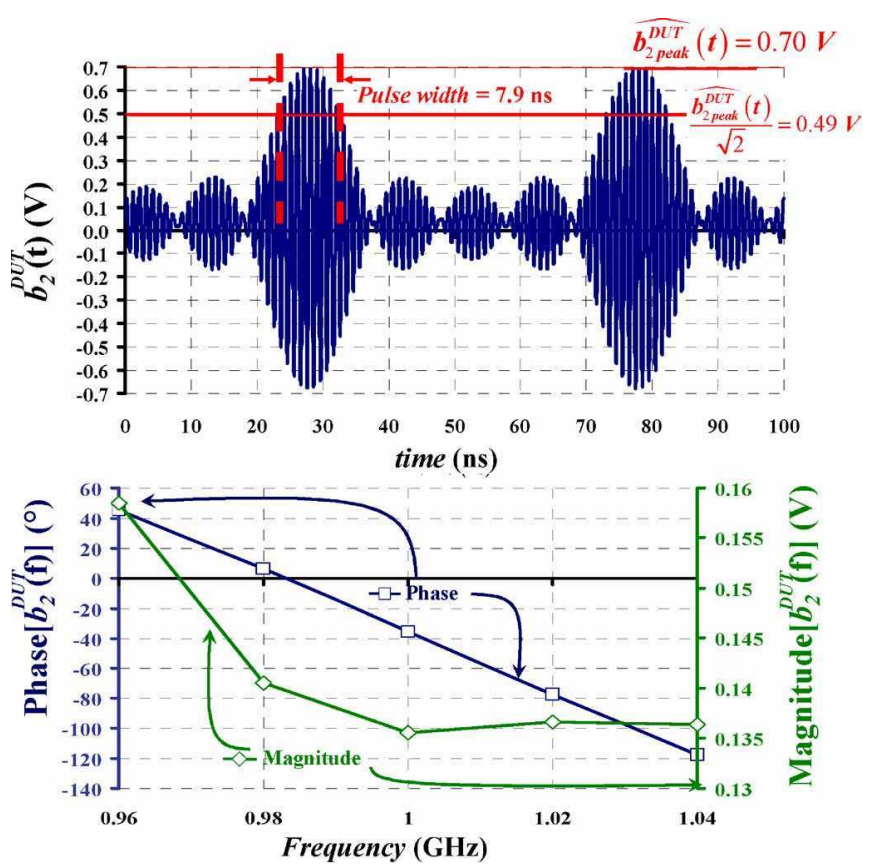

Fig. 25. Digital sampling oscilloscope measurements of a five-tone multisine (80-MHz bandwidth).
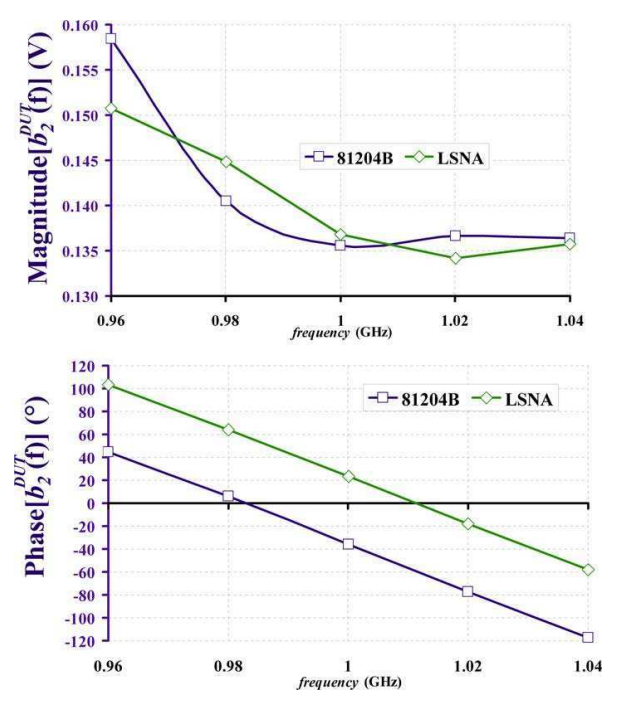

Fig. 26. Comparison of digital sampling oscilloscope and LSNA measurements of a five-tone multisine.

LSNA measurements and oscilloscope measurements. If one removes this constant phase offset, one obtains the phase difference between the five tones versus the frequency plotted in Fig. 27.

For the LSNA measurements, a $1.8^{\circ}$ maximum phase variation is observed around a mean value of $-40.25^{\circ}$, while a $3.1^{\circ}$ maximum phase variation is observed around a mean value of $-40.6^{\circ}$ for digital sampling oscilloscope measurements. This comparison between the direct oscilloscope measurements and the measurements performed with the LSNA calibrated with the new HPR generator demonstrates the effectiveness of the approach proposed in this paper to extract waveforms of wideband multisines.

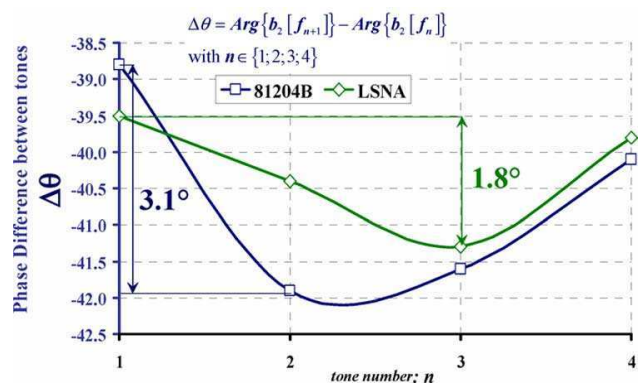

Fig. 27. Phase difference between the five tones measured with the digital sampling scope and the LSNA.

\section{CONCLUSION}

A fully calibrated measurement technique that enables phase and magnitude measurements of wideband multisines has been presented in this paper. This measurement technique is based on the use of an LSNA with a phase-calibration procedure using a new standard calibration. This technique has been compared to a direct digital sampling oscilloscope measurement. The effectiveness of the LSNA measurement technique has been demonstrated.

20- or $40-\mathrm{MHz}$ frequency grid multisines have been characterized and used as test signals as they correspond to channel frequency spacing in some satellite payloads.

The measurement principle has been validated for an $80-\mathrm{MHz}$ bandwidth signal at the $L$-band. It can be extended to 250 - or $500-\mathrm{MHz}$ bandwidths at the $K a$-band required for multipactor experiments in satellite payloads [27]. For that purpose, a very wideband source is needed in order to extend this study and to characterize the multipactor effect in OMUX satellite payloads.

Another topic of interest is the characterization of wideband high-power amplifiers in the order of $100 \mathrm{~W}$. In such high-power amplifiers, memory effects will be more pronounced and, as a consequence, they require accurate magnitude and phase asymmetry characterization of intermodulation products.

\section{ACKNOWLEDGMENT}

The research reported here was performed under the context of the Top Amplifier Research Groups in a European Team (TARGET) network.

The authors acknowledge the assistance of the Centre National d'Etudes Spatiales (CNES), Toulouse, France.

\section{REFERENCES}

[1] U. Lott, "Measurement of magnitude and phase of harmonics generated in nonlinear microwave two ports," IEEE Trans. Microw. Theory Tech., vol. 37 , no. 10 , pp. $1506-1511$, Oct. 1989.

[2] J. Verspecht, "Calibration of a measurement system for high frequency nonlinear devices," Ph.D. dissertation, Dept. ELEC., Vrije Univ. Brussel, Brussels, Belgium, 1995.

[3] J. Verspecht and K. Rush, "Individual characterization of broadband sampling oscilloscopes with a 'nose to nose' calibration procedure," IEEE Trans. Instrum. Meas., vol. IM-43, no. 2, pp. 347-354, Apr. 1994.

[4] D. F. Williams, P. D. Hale, T. S. Clement, and J. M. Morgan, "Calibrating electro-optic sampling systems," in IEEE MTT-S Int. Microw. Symp. Dig., Phoenix, AZ, May 20-25, 2001, pp. 1527-1530. 
[5] J.-M. Nebus, A. Mallet, D. Barataud, F. Blache, J.-P. Villotte, M. V. Bossche, and J. Verspecht, "Optimization of power added efficiency of transistors using the combination of an active harmonic load-pull setup with a broadband vectorial nonlinear network analyzer," in IEEE MTT-S Int. Microw. Symp. Workshop, San Francisco, CA, 1996, pp. 1365-1368, Paper TH2 B3.

[6] P. J. Tasker, "Non-linear vector network analyser NLVNA," in IEE Conf. Pub., 2005, pp. 213-233.

[7] D. Barataud, C. Arnaud, B. Thibaud, M. Campovecchio, J.-M. Nebus, and J.-P. Villotte, "Measurements of time-domain voltage/current waveforms at RF and microwave frequencies based on the use of a vector network analyzer for the characterization of nonlinear devices-Application to high-efficiency power amplifiers and frequency-multipliers optimization," IEEE Trans. Instrum. Meas., vol. 47, no. 5, pp. $1259-1264$, Oct. 1998.

[8] C. J. Wei, Y. A. Tkachenko, J. C. M. Hwang, K. R. Smith, and A. H. Peake, "Internal-node waveform analysis of MMIC power amplifier," IEEE Trans. Microw. Theory Tech., vol. 43, no. 12, pp. 3037-3042, Dec. 1995.

[9] J. C. M. Hwang, "Internal waveform probing of HBT and HEMT MMIC power amplifiers," in 60th ARFTG Conf. Dig., Washington, DC, Dec. 5-6, 2002, pp. 111-112.

[10] T. Reveyrand, A. Mallet, J.-M. Nebus, and M. V. Bossche, "Calibrated measurements of waveforms at internal nodes of MMICs with a LSNA and high impedance probes," in 62nd ARFTG Microw. Meas. Conf., Dec. 4-5, 2003, pp. 71-76.

[11] F. De Groote, J. Verspecht, C. Tsironis, D. Barataud, and J.-P. Teyssier, "An improved coupling method for time-domain load-pull measurements," in 65th ARFTG Microw. Meas. Conf. Dig., 2005, pp. 53-56, Paper 1500568.

[12] W. Van Moer and Y. Rolain, "Proving the usefulness of a three-port nonlinear vectorial network analyzer through mixer measurements," IEEE Trans. Instrum. Meas., vol. 52, no. 6, pp. 1834-1837, Dec. 2003.

[13] A. Cidronali, K. C. Gupta, J. Jargon, K. A. Remley, D. DeGroot, and G. Manes, "Extraction of conversion matrices for P-HEMTs based on vectorial large-signal measurements," in IEEE MTT-S Int. Microw. Symp. Dig., 2003, vol. 2, pp. 777-780.

[14] Y. Yang, J. Yi, J. Nam, B. Kim, and M. Park, "Measurement of two-tone transfer characteristics of high-power amplifiers," IEEE Trans. Microw. Theory Tech., vol. 49, no. 3, pp. 568-571, Mar. 2001.

[15] P. Roblin, S. J. Doo, X. Cui, G. H. Jessen, D. Chaillot, and J. Strahler, "New ultra-fast real-time active load-pull measurements for high speed RF power amplifier design," in IEEE MTT-S Int. Microw. Symp. Dig., Honolulu, HI, Jun. 2007, pp. 1493-1496.

[16] K. A. Remley, D. M. M.-P. Schreurs, D. F. Williams, and J. Wood, "Extended NVNA bandwidth for long-term memory measurements," in IEEE MTT-S Int. Microw. Symp. Dig., Fort Worth, TX, Jun. 2004, vol. 3, pp. 1739-1742.

[17] A. Barel and Y. Rolain, "Microwave multisine with known phase for the calibration of narrowbanded nonlinear vectorial network analyzer measurements," in IEEE MTT-S Int. Microw. Symp. Dig., 1998, vol. 3 , pp. 1499-1502.

[18] J. Schoukens, Y. Rolain, and P. Guillaume, "Design of narrowband, high-resolution multisines," IEEE Trans. Instrum. Meas., vol. 45, no. 3, pp. 750-753, Jun. 1996.

[19] D. Schreurs and K. Remley, "Bandwidth extension using stitching approach," in ARFTG Nonlinear Meas. Workshop, Washington, DC, Nov. 30, 2005, 6 pp.

[20] M. El Yaagoubi, G. Neveux, D. Barataud, J.-M. Nebus, and J. Verspecht, "Accurate phase measurements of broadband multitone signals using a specific configuration of a large-signal network analyzer," in IEEE MTT-S Int. Microw. Symp. Dig., Jun. 2006, pp. 1448-1451.

[21] P. Blockley, D. Gunyan, and J. B. Scott, "Wide-bandwidth, vector-corrected measurement with high spurious-free dynamic range," in ARFTG Conf. Dig., Jun. 17, 2005, 4 pp.

[22] P. Blockley, D. Gunyan, and J. B. Scott, "Mixer-based, vector-corrected, vector signal/network analyzer offering $300 \mathrm{kHz}-20 \mathrm{GHz}$ bandwidth and traceable phase response," in IEEE MTT-S Int. Microw. Symp. Dig., Jun. 12-17, 2005, 4 pp.

[23] W. Van Moer and Y. Rolain, "An improved broadband conversion scheme for the large-signal network analyzer," in IEEE MTT-S Int. Microw. Symp. Dig., 2005, vol. 2005, pp. 1501-1504, Paper 1516978.

[24] T. Souders, D. Flach, C. Hagwood, and G. Yang, "The effects of timing jitter in sampling systems," in 6th IEEE Instrum. Meas. Technol. Conf. Rec., Apr. 25-27, 1989, pp. 199-203.
[25] G. Vandersteen, Y. Rolain, and J. Schoukens, "An identification technique for data acquisition characterization in the presence of nonlinear distortions and time base distortions," IEEE Trans. Instrum. Meas., vol. 50, no. 5, pp. 1355-1363, Oct. 2001.

[26] Y. Rolain, J. Schoukens, and G. Vandersteen, "Signal reconstruction for non-equidistant finite length sample sets: A 'KIS' approach,' IEEE Trans. Instrum. Meas., vol. 47, no. 5, pp. 1046-1052, Oct. 1998.

[27] J. Puech, L. Lapierre, J. Sombrin, V. Semenov, A. Sazontov, N. Vdovicheva, M. Buyanova, U. Jordan, R. Udiljak, D. Anderson, and M. Lisak, "Multipactor threshold in waveguides : Theory and experiment," in NATO Adv. Res. Workshop, Nizhny, Novgorod, Russian Federation, Feb. 17-20, 2004, pp. 305-323.

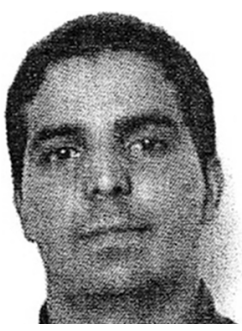

Mohammed El Yaagoubi was born in Taza, Morocco, on April 14, 1977. He received the Ph.D. degree in electronics from the University of Limoges, Limoges, France, in 2006.

$\mathrm{He}$ is currently with the University of Limoges, XLIM, Unité Mixte de Recherche (UMR).

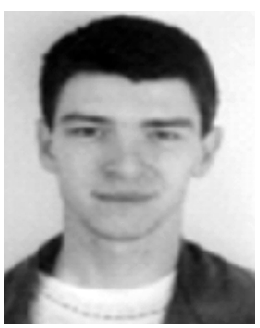

Guillaume Neveux was born in Civray, France, in 1976. He received the Diplôme d'Etudes Approfondies (DEA) degree from the Université Paris 11, Orsay, France, in 2000, and the Ph.D. degree in electronics and communications from the National Superior Institute of Telecommunications (ENST), Paris, France, in 2003.

Since 2004, he has been with the Instrumentation Group, XLIM, University of Limoges, Limoges, France. His research interests include nonlinear measurement with LSNAs and the study of RF

sampling systems.

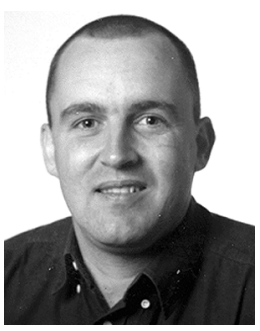

Denis Barataud was born in Saint-Junien, France, in 1970. He graduated from the Ecole Nationale Superieure de Telecommunications de Bretagne, Bretagne, France, in 1994. He received the Ph.D. degree in electronics from the University of Limoges, Liomges, France, in 1998.

From 1998 to 1999, he was a Post-Doctoral Scientist with the Microwave Laboratory, Centre National d'Etudes Spatiales (CNES), Toulouse, France. Since 2000, he has been with the XLIM [formerly the Institut de Recherche en Communications Optiques et Microondes (IRCOM)], University of Limoges, Limoges, France, where he became an Assistant Professor in 2001. His research interests include the development of time-domain equipment and techniques for the characterization of nonlinear devices.

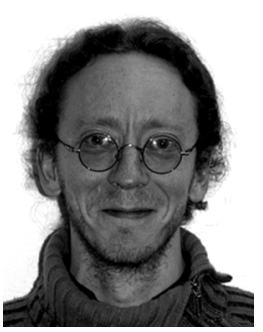

Tibault Reveyrand was born in Paris, France, on September 20, 1974. He received the Ph.D. degree from the University of Limoges, Limoges, France, in 2002.

From 2002 to 2004, he was a Post-Doctoral Scientist with the Centre National d'Etudes Spatiales (CNES) (French Space Agency). In 2005, he became a Contractual Center National de la Recherche Scientifique (CNRS) Engineer with the XLIM [formerly the Institut de Recherche en Communications Optiques et Microondes (IRCOM)], Limoges, France. His research interests include the characterization and modeling of RF and microwave nonlinear components.

Dr. Reveyrand was the recipient of the 2002 European GaAs Best Paper Award. 


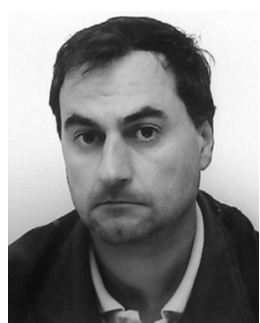

Jean-Michel Nebus was born in Bourganeuf, France in 1963. He received the Ph.D. degree in electronics from the University of Limoges, Limoges, France in 1988

He was a Project Engineer with ALCATEL Space Industries France. He is currently a Professor with the XLIM [formerly the Institut de Recherche en Communications Optiques et Microondes (IRCOM)], University of Limoges. His main area of interest is nonlinear microwave device characterization and design.

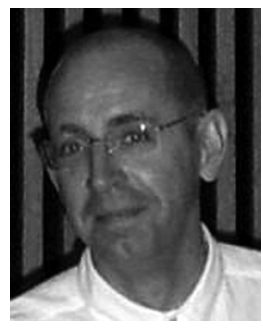

Frans Verbeyst (M'07) was born in Merchtem, Belgium, in 1963. He received the Electrotechnical-Mechanical Engineer degree from the Vrije Universiteit Brussel (VUB), Brussels, Belgium, in 1986, and the Werktuigkundig Elektrotechnisch Burgerliijk Ingenieur and Doctor in Engineering Sciences degrees from the Vrije Universiteit Brussel, Brussels, Belgium, in 1986 and 2007, respectively.

After four years with Philips (1990-2003), he joined NMDG, Agilent Technologies (HewlettPackard). Since he joined Hewlett-Packard/Agilent Technologies, he has been involved in large-signal RF and microwave measurements and behavioral modeling. He has also been involved with a streamlined implementation of the "nose-to-nose" calibration procedure as an essential part of the phase calibration of LSNAs and its transfer into other groups within Agilent Technologies and into the National Institute of Standards and Technology (NIST). Since 2003, he has been with NMDG Engineering BVBA (now NMDG NV), Bornem, Belgium. From July 2004 to June 2006, he worked part-time with the Vakgroep ELEC, Faculteit Ingenieurswetenschappen, Vrije
Universiteit Brussel, Brussels, Belgium, during completion of his doctoral dissertation "Contributions to Large-Signal Network Analysis."

Dr. Verbeyst was a corecipient of the 2002 ARFTG Technology Award. He was the recipient of two ARFTG Best Paper Awards.

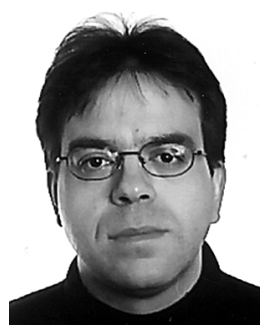

Francis Gizard was born in Cahors, France, in 1964. Since February 1988, he has been with the Microwave and Time-Frequency Department, Centre National d'Etudes Spatiales (CNES) (French Space Agency), Toulouse, France. His main areas of interest are linear and nonlinear microwave measurement techniques and on-wafer characterization.

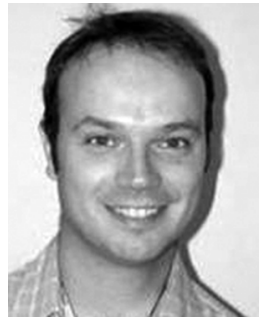

Jérôme Puech was born in Rodez, France, in 1974 He received the Dipl. Eng. degree from the Institut National des Télécommunications, Evry, France, in 1998.

In January 2000, he joined the Centre National d'Etudes Spatiales (CNES) (French Space Agency), Toulouse, France, where he is currently involved in microwave research activities that are mainly oriented toward microwave breakdown within space components, microwave filters, and traveling-wave tubes. 